\title{
High-Performance Ternary Organic Solar Cells Enabled by Synergizing Fullerene and Non-fullerene Acceptors
}

\author{
Yuanyuan Jiang ${ }^{\mathrm{a}, \mathrm{b}}$ (1) \\ Xiaozhang Zhu*a,b (i)

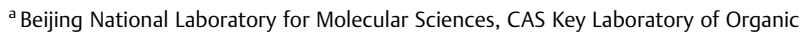 \\ Solids, Institute of Chemistry, Chinese Academy of Sciences, Beijing 100190, China \\ ${ }^{\mathrm{b}}$ School of Chemical Sciences, University of Chinese Academy of Sciences, Beijing \\ 100049, China \\ xzzhu@iccas.ac.cn
}

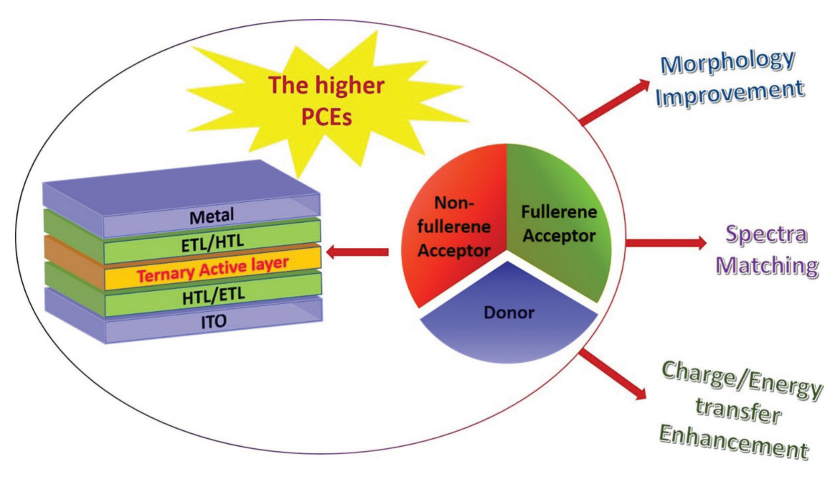

lightweight, flexibility, solution processability, and largescalability over the existing rigid and fragile inorganic solar cells. ${ }^{1}$ Over the past decades, many approaches have been used to promote the photovoltaic parameters of devices and to elevate power conversion efficiency (PCE), such as designing new materials, ${ }^{2-6}$ the introduction of additives, ${ }^{7}$ as well as the majorization of device structure ${ }^{8-10}$ and the electrode buffer layer. ${ }^{11,12}$

The PCE of OPVs is usually determined by the opencircuit voltage $\left(V_{\mathrm{oc}}\right)$, short-circuit current density $\left(J_{\mathrm{sc}}\right)$, and fill factor (FF): $\mathrm{PCE}=J_{\mathrm{sc}} \times V_{\mathrm{oc}} \times \mathrm{FF} / P_{\text {in }}$, where $P_{\text {in }}$ is the incident light power $\left(100 \mathrm{~mW} \mathrm{~cm}^{-2}\right.$ under illumination of AM $1.5 \mathrm{G}), J_{\mathrm{sc}}$ strongly depends on the number of absorbed photons as well as the probability of exciton dissociation and charge collection, and $V_{\text {oc }}$ relies on the energy difference between the LUMO of the acceptor and the HOMO of the donor. FF is mainly related to the competition between exciton dissociation and recombination in the active layer. The photovoltaic parameters are related to the thin-film morphology, such as phase separation, crystallinity, size or purity of domain, as well as the charge dynamics, which will be considered in the following. The morphology of the active layer is mainly determined by corresponding photovoltaic materials and can be optimized by various post-processing methods. And the morphology plays a critical role in device performance and stability. The ideal morphology usually presents the following features: (1) a proper phase separation with a suitable domain size, which can balance the competition between exciton dissociation and charge transport. Due to the limited exciton diffusion length $(10-20 \mathrm{~nm})$, the photoinduced excitons would not be dissociated effectively except for excitons generated near the donor/acceptor (D/A) interface. A large domain with a limited D/A interface causes insufficient exciton dissociation and an undesired low short-circuit current. A small domain is beneficial for exciton dissociation, but is disadvantageous for charge transport. ${ }^{13}$ (2) The bi- 

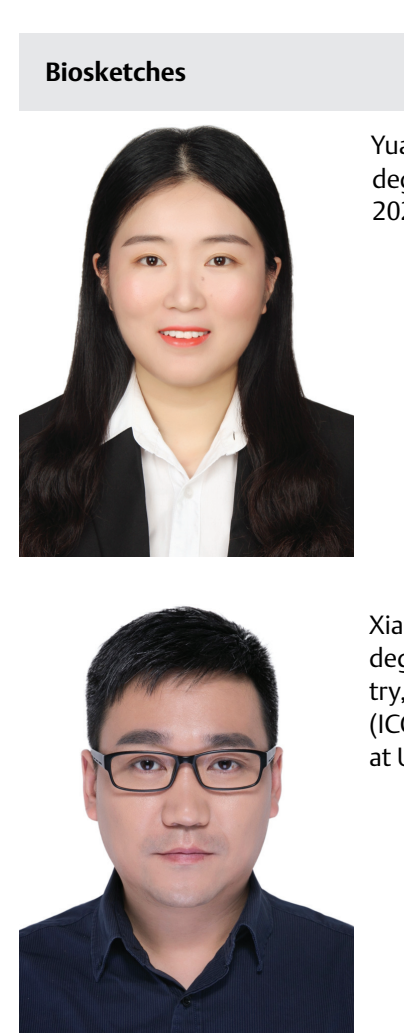

Xiaozhang Zhu received his Ph.D. degree from the Institute of Chemistry, Chinese Academy of Sciences (ICCAS), in 2006. Then, he worked at Ulm University as an Alexander von
Chemistry, Chinese Academy of Sciences, and is currently working toward her M.Sc. degree under the supervision of Prof. Xiaozhang Zhu. Her current research interest focuses on device fabrication of OPVs.
Humboldt research fellow and at the University of Tokyo as a Japan Society for the Promotion of Science research fellow from 2006 to 2012. He is currently a professor at ICCAS. His research is focused on organic $\pi-$ functional materials and their applications in organic optoelectronics. continuous interpenetrating networks that afford adequate transport channels for transporting holes and electrons to electrodes. ${ }^{14}$ (3) The phase purity that is closely related to the crystallinity of the electron donor and acceptor materials. The high phase purity favors the formation of good connectivity between the donor, acceptor and the anode, cathode, respectively, and thus facilitates charge collection; while the low phase purity may cause poor connectivity, which does not obstruct the charge transport but hinder charge transfer, leading to severe charge recombination. ${ }^{15,16}$ To achieve the ideal thin-film morphology, solvent additives are widely used in device fabrication; however, it shows disadvantages, such as being environmentally unfriendly and unstable. A ternary strategy of directly mixing three materials with complementary spectra in a ternary device also has an ability of morphology control. The additional component can fully utilize the solar spectrum and favor form cascade energy alignment or energy transfer. To sum up, the role of the third component in the development of ternary OPVs may be multiple, and it is inevitable that the ternary OPVs should be combined with post-processing and other strategies for optimal device performance. ${ }^{17-23}$ The ternary system involves the twodonor and the two-acceptor system. The two-acceptor system includes the options of fullerene-fullerene, fullerene-non-fullerene, and non-fullerene-non-fullerene. A fullerene acceptor (FA) exhibits excellent charge transport properties but suffers weak absorption. A non-FA (NFA) possesses an adjustable energy level as well as enhanced absorption compared with the FA, but sometimes shows poor thin-film morphology and low electron mobility. The matter of how to balance the various factors that affect the photovoltaic parameters to achieve a maximum PCE value via ternary OPVs will be discussed in this review.

Fullerene derivative acceptors have attracted much attention by right of its irreplaceable advantages for a long time ${ }^{24}$ : (1) the existence of low-lying $(0.2-0.4 \mathrm{eV})$ excited states in fullerene anions is responsible for the efficient charge separation at the D/A interface; (2) the high electron mobility; (3) a favorable nanoscale morphology; (4) an outstanding electron transport, the unique threedimensional (3D) structure would afford more electron channels than the two or one dimensions. In previous works, the research studies of OPVs were mainly concentrated on the design and synthesis of low-bandgap donor materials with broad absorption and suitable energy levels to match FAs. However, FAs show disadvantages such as limited absorption windows, insufficient long-term device stability, and relatively high cost, which may hinder further improvement of device performance and thus the progress of the commercialization of OPVs. Therefore, the emergence of NFAs as desired substitutes for the FAs is reasonable and inevitable. ${ }^{25}$ In contrast to the FAs, NFAs show unique advantages: (1) NFAs exhibit a broader absorption and a higher absorption coefficient than FAs. A broad or complementary absorption can be obtained by regulating the 
Table 1 Photovoltaic parameters of ternary OPV containing non-fullerene as the host acceptor $\left(A_{1}\right)$ and fullerene as the guest acceptor $\left(A_{2}\right)$ as well as non-fullerene-based binary OPV

\begin{tabular}{|c|c|c|c|c|c|c|c|c|c|c|}
\hline \multirow[b]{2}{*}{ Binary blend } & \multicolumn{5}{|c|}{ Photovoltaic parameters (binary) } & \multicolumn{5}{|c|}{ Photovoltaic parameters (ternary) } \\
\hline & $J_{\mathrm{sC}}\left(\mathrm{mA} \mathrm{cm} \mathrm{cm}^{-2}\right)$ & $V_{\mathrm{OC}}(\mathrm{V})$ & $\mathrm{FF}(\%)$ & PCE (\%) & $\mathrm{A}_{2}$ & $J_{\mathrm{SC}}\left(\mathrm{mA} \mathrm{cm} \mathrm{cm}^{-2}\right)$ & $V_{\mathrm{OC}}(\mathrm{V})$ & $\mathrm{FF}(\%)$ & PCE $(\%)$ & Ref. \\
\hline PM6: BTCT-2Cl & 24.4 & 0.877 & 70.4 & 15.1 & $\mathrm{PC}_{71} \mathrm{BM}$ & 25.1 & 0.881 & 72.6 & 16.1 & 20 \\
\hline P2F-EHp: Y6 & 25.27 & 0.78 & 71.1 & 14.16 & $\mathrm{PC}_{61} \mathrm{BM}$ & 26.38 & 0.82 & 74.8 & 16.18 & 35 \\
\hline BTR-Cl: Y6 & 23.79 & 0.8334 & 69.63 & 13.81 & $\mathrm{PC}_{71} \mathrm{BM}$ & 23.75 & 0.8378 & 77.11 & 15.34 & 38 \\
\hline PCE10: 3TT-OCIC & 26.49 & 0.68 & 69 & 12.43 & $\mathrm{PC}_{71} \mathrm{BM}$ & 27.58 & 0.69 & 69 & 13.13 & 41 \\
\hline PM6: Y18 & 25.71 & 0.84 & 76.5 & 16.52 & $\mathrm{PC}_{71} \mathrm{BM}$ & 26.3 & 0.84 & 77.4 & 17.11 & 42 \\
\hline PBDB-T: INPIC-Si & 19.80 & 0.901 & 66.09 & 11.79 & $\mathrm{PC}_{71} \mathrm{BM}$ & 20.98 & 0.892 & 70.84 & 13.26 & 43 \\
\hline PPBDTBT: ITIC & 13.00 & 0.925 & 63 & 7.72 & $\mathrm{PC}_{71} \mathrm{BM}$ & 16.66 & 0.894 & 68 & 10.41 & 44 \\
\hline PTFBDT-BZS: ITIC & 12.54 & 0.983 & 56 & 6.97 & $\mathrm{PC}_{71} \mathrm{BM}$ & 15.63 & 0.932 & 63 & 9.25 & 45 \\
\hline PBDB-T: IDT-2O & 15.70 & 0.86 & 71.60 & 9.65 & $\mathrm{PC}_{71} \mathrm{BM}$ & 16.80 & 0.87 & 72.11 & 10.67 & 46 \\
\hline PBDB-T: IDT-EDOT & 18.42 & 0.87 & 62.3 & 9.93 & $\mathrm{PC}_{71} \mathrm{BM}$ & 20.84 & 0.88 & 66.7 & 12.07 & 48 \\
\hline DR3TSBDT: Y6 & 21.67 & 0.879 & 55.21 & 10.53 & $\mathrm{PC}_{71} \mathrm{BM}$ & 22.19 & 0.858 & 67.27 & 12.84 & 49 \\
\hline PTQ10: Y6 & 24.64 & 0.851 & 71.67 & 15.03 & $\mathrm{PC}_{71} \mathrm{BM}$ & 25.32 & 0.850 & 74.69 & 16.07 & 50 \\
\hline PBDB-T: IOTC & 15.04 & 0.95 & 55.52 & 7.94 & $\mathrm{PC}_{71} \mathrm{BM}$ & 16.35 & 0.93 & 64.90 & 9.85 & 51 \\
\hline PBDB-T: ITIC & 15.06 & 0.902 & 69 & 9.38 & $\mathrm{PC}_{71} \mathrm{BM}$ & 15.98 & 0.892 & 71.7 & 10.22 & 52 \\
\hline PBDTTT-E-T: IEICO & 17.31 & 0.83 & 58 & 8.33 & Bis- $\mathrm{PC}_{70} \mathrm{BM}$ & 18.92 & 0.83 & 65 & 10.21 & 53 \\
\hline PBT1-C: ITIC-2CI & 18.03 & 0.86 & 70.3 & 11.10 & ICBA & 19.30 & 0.89 & 75.9 & 13.40 & 54 \\
\hline P3TCO-1: ITIC & 16.96 & 0.943 & 62.82 & 10.11 & $\mathrm{PC}_{71} \mathrm{BM}$ & 18.05 & 0.939 & 66.67 & 11.41 & 55 \\
\hline PBDTTPD-HT: ITIC & 14.86 & 0.97 & 69 & 9.95 & $\mathrm{PC}_{71} \mathrm{BM}$ & 17.38 & 0.95 & 74 & 12.09 & 56 \\
\hline PBT1-C: IT-2F & 17.28 & 0.879 & 72.7 & 11.04 & $\mathrm{PC}_{71} \mathrm{BM}$ & 18.19 & 0.892 & 75.1 & 12.19 & 57 \\
\hline PM6: Y6 & 24.89 & 0.845 & 74.37 & 15.75 & $\mathrm{PC}_{71} \mathrm{BM}$ & 25.70 & 0.85 & 76.35 & 16.67 & 58 \\
\hline PTB7-Th: F8IC & 25.12 & 0.640 & 67.6 & 10.90 & $\mathrm{PC}_{71} \mathrm{BM}$ & 25.61 & 0.670 & 71.5 & 12.30 & 59 \\
\hline PBDB-T: BDTC-4CI & 18.56 & 0.864 & 59.5 & 9.54 & $\mathrm{PC}_{71} \mathrm{BM}$ & 21.19 & 0.856 & 67.2 & 12.19 & 60 \\
\hline PTBTz-2: ITIC & 19.8 & 0.89 & 59.05 & 10.43 & $\mathrm{PC}_{71} \mathrm{BM}$ & 20.75 & 0.89 & 60.94 & 11.26 & 61 \\
\hline PTBTz-2: IT-M & 17.61 & 0.954 & 61.84 & 10.39 & $\mathrm{PC}_{71} \mathrm{BM}$ & 18.70 & 0.928 & 70.78 & 12.28 & 62 \\
\hline PM6: BTP-4Cl & 25.49 & 0.868 & 72.21 & 15.98 & ICBA & 26.13 & 0.876 & 73.23 & 16.76 & 63 \\
\hline POBDFBT: ITIC & 16.59 & 0.82 & 46.5 & 6.16 & $\mathrm{PC}_{71} \mathrm{BM}$ & 17.65 & 0.72 & 61.8 & 7.91 & 64 \\
\hline PBDTNS-BDD: ITIC & 14.86 & 0.94 & 66.47 & 9.28 & $\mathrm{PC}_{71} \mathrm{BM}$ & 17.77 & 0.93 & 67.81 & 11.21 & 65 \\
\hline PTB7: 3TT-FIC & 25.89 & 0.662 & 71.2 & 12.21 & $\mathrm{PC}_{71} \mathrm{BM}$ & 27.73 & 0.669 & 73.0 & 13.54 & 66 \\
\hline PTB7-Th: IFIC-i-4F & 22.90 & 0.65 & 63 & 10.03 & $\mathrm{PC}_{71} \mathrm{BM}$ & 24.03 & 0.67 & 68 & 11.49 & 67 \\
\hline SBDT-BDD: IDIC & 15.15 & 0.97 & 62.5 & 9.20 & $\mathrm{PC}_{71} \mathrm{BM}$ & 16.21 & 0.97 & 69.3 & 10.90 & 68 \\
\hline PBDB-T: IT-M & 16.7 & 0.937 & 69.0 & 10.80 & Bis- $\mathrm{PC}_{70} \mathrm{BM}$ & 17.39 & 0.952 & 73.7 & 12.20 & 69 \\
\hline PBDB-T: ITIC & 15.9 & 0.92 & 65.6 & 9.60 & $\mathrm{PC}_{71} \mathrm{BM}$ & 17.7 & 0.91 & 68.3 & 11.00 & 72 \\
\hline PTB7-Th: IEICO-4F & 21.3 & 0.73 & 60 & 9.44 & $\mathrm{PC}_{71} \mathrm{BM}$ & 22.64 & 0.74 & 63 & 10.55 & 73 \\
\hline $\mathrm{P}(\mathrm{Cl}-\mathrm{Cl})(\mathrm{BDD}=0.2): \mathrm{IT}-4 \mathrm{~F}$ & 20.0 & 0.878 & 69.1 & 12.10 & $\mathrm{PC}_{71} \mathrm{BM}$ & 21.2 & 0.878 & 70.7 & 13.20 & 74 \\
\hline PM6: Y6 & 24.5 & 0.848 & 74.6 & 15.50 & $\mathrm{PC}_{71} \mathrm{BM}$ & 25.1 & 0.861 & 77.2 & 16.7 & 75 \\
\hline PBDB-T: IPIC-4CI & 22.2 & 0.813 & 74.0 & 13.40 & $\mathrm{PC}_{71} \mathrm{BM}$ & 23.3 & 0.822 & 74.6 & 14.30 & 76 \\
\hline PDBT-F: IDIC & 17.65 & 0.87 & 71.50 & 11.02 & $\mathrm{PC}_{71} \mathrm{BM}$ & 18.69 & 0.89 & 70.88 & 11.86 & 77 \\
\hline DR3: ICC6 & 15.2 & 0.87 & 64 & 8.80 & $\mathrm{PC}_{71} \mathrm{BM}$ & 16.3 & 0.87 & 72 & 10.80 & 78 \\
\hline PTB7-Th: $\mathrm{CO}_{\mathrm{i}} 8 \mathrm{DFIC}$ & 23.84 & 0.69 & 63.8 & 10.48 & $\mathrm{PC}_{71} \mathrm{BM}$ & 28.20 & 0.70 & 71 & 14.08 & 79 \\
\hline PM6: N3 & 25.81 & 0.837 & 73.9 & 15.98 & $\mathrm{PC}_{71} \mathrm{BM}$ & 25.71 & 0.85 & 76.6 & 16.74 & 80 \\
\hline PBDB-T: ITIC & 15.44 & 0.903 & 67.7 & 9.76 & $\mathrm{PC}_{71} \mathrm{BM}$ & 17.84 & 0.882 & 69.8 & 11.22 & 81 \\
\hline PM6: ITC6-4F & 20.12 & 0.90 & 74.11 & 13.42 & $\mathrm{PC}_{61} \mathrm{BM}$ & 20.78 & 0.93 & 78.18 & 15.11 & 82 \\
\hline SM1: MPU4 & 14.91 & 0.90 & 64 & 8.96 & $\mathrm{PC}_{71} \mathrm{BM}$ & 16.32 & 0.92 & 67 & 10.05 & 83 \\
\hline
\end{tabular}


Table 1 (Continued)

Photovoltaic parameters (binary)

Photovoltaic parameters (ternary)

\begin{tabular}{|c|c|c|c|c|c|c|c|c|c|c|}
\hline Binary blend & $J_{\mathrm{sC}}\left(\mathrm{mA} \mathrm{cm}{ }^{-2}\right)$ & $V_{\mathrm{OC}}(\mathrm{V})$ & $\mathrm{FF}(\%)$ & PCE (\%) & $\mathrm{A}_{2}$ & $J_{\mathrm{sC}}\left(\mathrm{mA} \mathrm{cm}{ }^{-2}\right)$ & $V_{\mathrm{OC}}(\mathrm{V})$ & $\mathrm{FF}(\%)$ & PCE (\%) & Ref. \\
\hline POTz: IDIC & 16.32 & 0.82 & 63.71 & 8.52 & $\mathrm{PC}_{70} \mathrm{BM}$ & 17.47 & 0.82 & 65.14 & 9.33 & 84 \\
\hline PBDB-T: IDTC-4CI & 19.19 & 0.822 & 60.2 & 9.50 & $\mathrm{PC}_{71} \mathrm{BM}$ & 19.14 & 0.829 & 65.6 & 10.41 & 85 \\
\hline J52: IEICO-4F & 22.27 & 0.675 & 61.3 & 9.21 & $\mathrm{PC}_{71} \mathrm{BM}$ & 22.70 & 0.698 & 67.4 & 10.68 & 87 \\
\hline PTB7-Th: IEICO-4F & 20.62 & 0.74 & 58.55 & 9.32 & $\mathrm{PC}_{71} \mathrm{BM}$ & 23.63 & 0.74 & 58.00 & 10.63 & 88 \\
\hline PBDB-T: ITIC & 16.00 & 0.89 & 65 & 9.03 & $\mathrm{PC}_{71} \mathrm{BM}$ & 17.45 & 0.89 & 68 & 10.43 & 89 \\
\hline DR3: ICC6 & 15.2 & 0.87 & 64 & 8.70 & $\mathrm{PC}_{71} \mathrm{BM}$ & 16.3 & 0.87 & 72 & 10.80 & 91 \\
\hline PBDB-T: DTFT9-FIC & 19.01 & 0.86 & 58.61 & 9.58 & $\mathrm{PC}_{71} \mathrm{BM}$ & 20.59 & 0.88 & 65.27 & 11.82 & 94 \\
\hline PM6: FCTT-FIC & 19.49 & 0.90 & 69.73 & 12.23 & $\mathrm{PC}_{71} \mathrm{BM}$ & 19.86 & 0.92 & 73.20 & 13.37 & 95 \\
\hline PM6: Y6-C2 & 25.11 & 0.860 & 73.6 & 15.89 & $\mathrm{PC}_{71} \mathrm{BM}$ & 25.73 & 0.859 & 77.2 & 17.06 & 96 \\
\hline PBDB-TF: Y6 & 24.8 & 0.834 & 74.1 & 15.30 & $\mathrm{PC}_{61} \mathrm{BM}$ & 25.4 & 0.845 & 77.0 & 16.50 & 97 \\
\hline PBDB-T-2Cl: BTP-4F & 26.2 & 0.854 & 70.1 & 15.70 & $\mathrm{PC}_{61} \mathrm{BM}$ & 26.4 & 0.842 & 73.3 & 16.3 & 98 \\
\hline PBDB-T: BDCPDT-IC & 16.56 & 0.86 & 65.52 & 9.33 & $\mathrm{PC}_{71} \mathrm{BM}$ & 16.84 & 0.84 & 68.79 & 9.73 & 100 \\
\hline PBDB-T: IEICO-4F & 20.45 & 0.74 & 65.2 & 9.87 & $\mathrm{NC}_{70} \mathrm{BA}$ & 20.49 & 0.81 & 65.8 & 10.92 & 101 \\
\hline CS01: MPU3 & 13.04 & 1.07 & 0.56 & 7.81 & $\mathrm{PC}_{71} \mathrm{BM}$ & 16.27 & 0.97 & 63 & 9.94 & 102 \\
\hline PTB7Th: $\mathrm{CO}_{\mathrm{i}} 8 \mathrm{DFIC}$ & 22.96 & 0.704 & 66.80 & 10.80 & $\mathrm{PC}_{61} \mathrm{BM}$ & 27.01 & 0.718 & 73.34 & 14.22 & 105 \\
\hline PMTT56: IT-2F & 18.67 & 0.945 & 71.4 & 12.60 & $\mathrm{PC}_{71} \mathrm{BM}$ & 19.75 & 0.932 & 71.4 & 13.20 & 104 \\
\hline PBDB-TF: HC-PCIC & 17.54 & 0.88 & 72.69 & 11.48 & $\mathrm{PC}_{71} \mathrm{BM}$ & 19.29 & 0.89 & 70.18 & 12.36 & 106 \\
\hline PM6: IT-4F & 20.14 & 0.857 & 71.42 & 12.33 & ICBA & 20.64 & 0.871 & 73.30 & 13.70 & 107 \\
\hline PBDTTT-OFT: IEICO-4F & 24.7 & 0.71 & 68 & 11.90 & $\mathrm{PC}_{71} \mathrm{BM}$ & 26.1 & 0.72 & 69 & 13.00 & 108 \\
\hline PFBDT-8ttTPD: Y6 & 24.99 & 0.84 & 72 & 15.05 & $\mathrm{PC}_{71} \mathrm{BM}$ & 25.96 & 0.85 & 74 & 16.43 & 110 \\
\hline PM6: N3 & 25.4 & 0.835 & 73.5 & 15.6 & $\mathrm{PC}_{71} \mathrm{BM}$ & 26.0 & 0.840 & 76.6 & 16.7 & 145 \\
\hline
\end{tabular}

bandgap of NFAs. ${ }^{26,27}$ The D/A approach, first reported by Havinga's group, ${ }^{28,29}$ is widely used in shrinking the bandgap of NFAs with absorption extending to nearinfrared, where $D$ is the electron-donating unit and has strong ties with the HOMO energy level, and A is the electron-withdrawing unit and is bound up with the LUMO energy level. Moreover, this approach can independently mediate the HOMO or LUMO energy levels. It might be an easy method for modulating the optoelectronic properties. Much research studies have been conducted to shrink the bandgap of the NFAs for a larger overlap with the solar spectrum, such as strengthening the electron-donating capability of the donor (D) unit to lift the HOMO energy level, ${ }^{30-32}$ or weakening the electron-withdrawing ability of the acceptor (A) unit to lower the LUMO energy level. ${ }^{33}$ (2) The tunable energy level of non-fullerenes that can be achieved by sensible adjusting of the molecular structure to better match the donor material, thus to improve $V_{\text {oc }}$ (3) NFAs show a relatively low voltage loss. (4) NFAs have a relatively low cost and are easy to purify, whereas NFAbased binary OPVs usually suffer poor thin-film morphology and charge dynamics. Therefore, the promising ternary strategy (containing three photovoltaic materials in a device) of using fullerenes and non-fullerenes as acceptors can be a simple way of overcoming the shortcomings of FAor NFA-based binary OPVs and preserving their advantages for optimal device performance. ${ }^{34}$ Besides, ternary OPVs also show their potential in large-scale devices for commercialization. $^{35}$

A previous work includes a systematic and detailed summary of the ternary OPV from different aspects. ${ }^{36}$ As for the change of key photovoltaic parameters, $V_{\mathrm{oc}}, J_{\mathrm{sc}}$ and $\mathrm{FF}$ as well as the function of the second acceptor in ternary OPVs based on FA and NFA will be emphasized in this report. Herein, we collected the photovoltaic parameters of ternary devices and corresponding binary devices (Tables 1 and 2), and the chemical structures of donors and acceptors are listed in Figures 1 and 2.

\section{Incorporation of Fullerene Derivatives into NFA-Based Binary Systems}

\section{Improving Blend Morphology}

As non-fullerene small molecules with a planar structure usually pack in one preferred orientation, which may be beneficial for charge transport, sometimes this may cause 
Table 2 Photovoltaic parameters of ternary OPV containing fullerene as the host acceptor $\left(A_{1}\right)$ and non-fullerene as the guest acceptor $\left(A_{2}\right)$ as well as fullerene-based binary OPV

\begin{tabular}{|c|c|c|c|c|c|c|c|c|c|c|}
\hline \multirow[b]{2}{*}{ Binary blend } & \multicolumn{5}{|c|}{ Photovoltaic parameters (binary) } & \multicolumn{5}{|c|}{ Photovoltaic parameters (ternary) } \\
\hline & $J_{\mathrm{sc}}\left(\mathrm{mA} \mathrm{cm}{ }^{-2}\right)$ & $V_{\mathrm{OC}}(\mathrm{V})$ & $\mathrm{FF}(\%)$ & PCE (\%) & $\mathrm{A}_{2}$ & $J_{\mathrm{sc}}\left(\mathrm{mA} \mathrm{cm}{ }^{-2}\right)$ & $V_{\mathrm{OC}}(\mathrm{V})$ & $\mathrm{FF}(\%)$ & PCE $(\%)$ & $R$ \\
\hline PffBT-DPP: PC 70 BM & 12.5 & 0.74 & 74 & 6.8 & MelC & 16.1 & 0.76 & 73 & 9.0 & 34 \\
\hline DRTB-T: PC ${ }_{71} B M$ & 9.67 & 0.99 & 65.3 & 6.23 & IDIC & 15.47 & 0.99 & 67.7 & 10.48 & 40 \\
\hline PBDTBDD: $\mathrm{PC}_{60} \mathrm{BM}$ & 10.99 & 0.87 & 69.19 & 6.63 & ITIC & 17.76 & 0.91 & 63.47 & 10.36 & 90 \\
\hline PBTTFB: $\mathrm{PC}_{70} \mathrm{BM}$ & 10.79 & 0.99 & 74 & 7.98 & ITIC & 13.73 & 1.01 & 71 & 9.73 & 92 \\
\hline PTB7-Th: PC $_{71}$ BM & 16.21 & 0.778 & 60.1 & 7.58 & CPDT-(TIC $)_{2}$ & 19.00 & 0.803 & 61.6 & 9.48 & \\
\hline РВTВT-ОTT: $\mathrm{PC}_{71} \mathrm{BM}$ & 14.43 & 0.92 & 59.1 & 7.83 & ITIC & 16.61 & 0.92 & 56.4 & 8.61 & \\
\hline PBTA-BO: PC $_{61}$ BM & 8.93 & 0.873 & 73.02 & 5.70 & IFBR & 13.45 & 0.926 & 65.07 & 8.11 & \\
\hline PPDT2FBT: $\mathrm{PC}_{71} \mathrm{BM}$ & 14.75 & 0.79 & 79 & 8.16 & IDT2BR & 15.50 & 0.82 & 71 & 9.02 & \\
\hline PBDTBDD: $\mathrm{PC}_{71} \mathrm{BM}$ & 14.19 & 0.84 & 66.18 & 7.86 & IEICO & 17.98 & 0.85 & 68.85 & 10.51 & \\
\hline PDOT: $\mathrm{PC}_{71} \mathrm{BM}$ & 13.92 & 0.94 & 72.9 & 9.54 & ITIC & 17.49 & 0.96 & 66.8 & 11.21 & \\
\hline PTB7-Th: PC ${ }_{71}$ BM & 17.39 & 0.80 & 65 & 9.16 & IEICO-4F & 23.68 & 0.77 & 61 & 11.24 & \\
\hline $\mathrm{P1}: \mathrm{PC}_{71} \mathrm{BM}$ & 12.60 & 0.90 & 70.64 & 8.07 & ITIC & 17.71 & 0.90 & 63.67 & 10.11 & \\
\hline PBT-OTT: $\mathrm{PC}_{71} \mathrm{BM}$ & 13.3 & 0.83 & 58.5 & 6.74 & ITIC & 14.8 & 0.87 & 63 & 8.18 & \\
\hline PM6: $\mathrm{PC}_{71} \mathrm{BM}$ & 12.59 & 0.92 & 63 & 7.38 & N2200-F & 13.01 & 0.91 & 69 & 8.11 & 1 \\
\hline PBTA-BO: $\mathrm{PC}_{71} \mathrm{BM}$ & 9.31 & 0.903 & 55.65 & 4.73 & IffBR & 14.52 & 0.908 & 67.47 & 9.06 & 126 \\
\hline PTB7-Th: PC $_{71}$ BM & 16.73 & 0.796 & 63.81 & 8.50 & IT-M & 17.82 & 0.820 & 67.54 & 9.87 & 127 \\
\hline PBDB-T: PC ${ }_{71} \mathrm{BM}$ & 14.66 & 0.81 & 61.65 & 7.25 & ITIC & 17.76 & 0.85 & 60.92 & 9.34 & 128 \\
\hline BTR: PC ${ }_{71} \mathrm{BM}$ & 13.80 & 0.90 & 72.86 & 9.03 & NITI & 19.50 & 0.94 & 73.83 & 13.64 & 12 \\
\hline PTB7-Th: PC $_{71}$ BM & 16.47 & 0.77 & 71.2 & 9.51 & TPE-4PDI & 17.44 & 0.78 & 73.9 & 10.40 & 130 \\
\hline PTB7-Th: PC $_{71}$ BM & 16.3 & 0.80 & 67.2 & 9.10 & IDIC & 18.7 & 0.79 & 69.6 & 10.70 & 132 \\
\hline PBDB-T: PC ${ }_{71} \mathrm{BM}$ & 11.40 & 0.89 & 67.8 & 6.98 & DTCFOIC & 18.46 & 0.87 & 63.3 & 10.13 & 133 \\
\hline PDBT-T1: $\mathrm{PC}_{70} \mathrm{BM}$ & 13.24 & 0.915 & 76.2 & 9.29 & ITIC-Th & 15.94 & 0.934 & 70.5 & 10.48 & 134 \\
\hline PTB7-Th: PC $_{71}$ BM & 18.43 & 0.79 & 64.56 & 9.69 & DIBC & 20.68 & 0.77 & 73.37 & 12.17 & 135 \\
\hline PTB7-Th: PC ${ }_{71}$ BM & 17.2 & 0.78 & 70 & 9.80 & TPB & 19.4 & 0.78 & 68 & 10.60 & 136 \\
\hline PTB7: $\mathrm{PC}_{71} \mathrm{BM}$ & 16.7 & 0.716 & 50.63 & 6.20 & IDFBR & 17.38 & 0.731 & 55.56 & 7.10 & \\
\hline
\end{tabular}

serious aggregation and thus recombination. ${ }^{37}$ The incorporation of a FA into a binary organic solar cell (OSC) based on NFA may help to form a favorable morphology in ternary OSCs and thus improve device performance. ${ }^{38}$ Therefore, numerous works have attempted to ameliorate the binary morphology via the ternary tactics, and the introduction of fullerene derivatives as the second acceptor was found to have the following effects. (1) A fine phase separation with a suitable domain size and an adequate $\mathrm{D} / \mathrm{A}$ interface is formed to balance the trade-off between exciton dissociation and charge transport. (2) The crystallinity of the NFAbased blend can be adjusted; the high material crystallinity presents excellent charge mobility and pure domains, and then facilitates the formation of a bi-continuous interpenetrating network structure for efficient charge transport. (3) An ideal vertical phase distribution can be formed, which can improve the charge generation, transport, and collection. In summary, the FA can act as a morphology regulator to modify the thin-film morphology in NFA-based binary OSCs.

Phase separation: The domain size has great influence on charge generation and transport. The components with good miscibility may form fine phase separation with small donor and acceptor domains, which can offer sufficient $\mathrm{D} / \mathrm{A}$ interface for exciton diffusion and dissociation; however, due to the discontinuous network and the limited carrier lifetime, the free charge carriers (holes and electrons) cannot be transported to the electrodes, leading to the undesired $J_{s c}$ and FF. On the other hand, in the blend with large phase separation, effective charge transport can be expected but the insufficient $\mathrm{D} / \mathrm{A}$ interface and the exciton diffusion length will lead to poor exciton dissociation. Thus, to guarantee promising charge generation and transport, the domain size should be well-controlled. NFAs with a planar core tend to form large aggregates, leading to a large phase and thus creating a poor FF in NFA-based binary cells. 

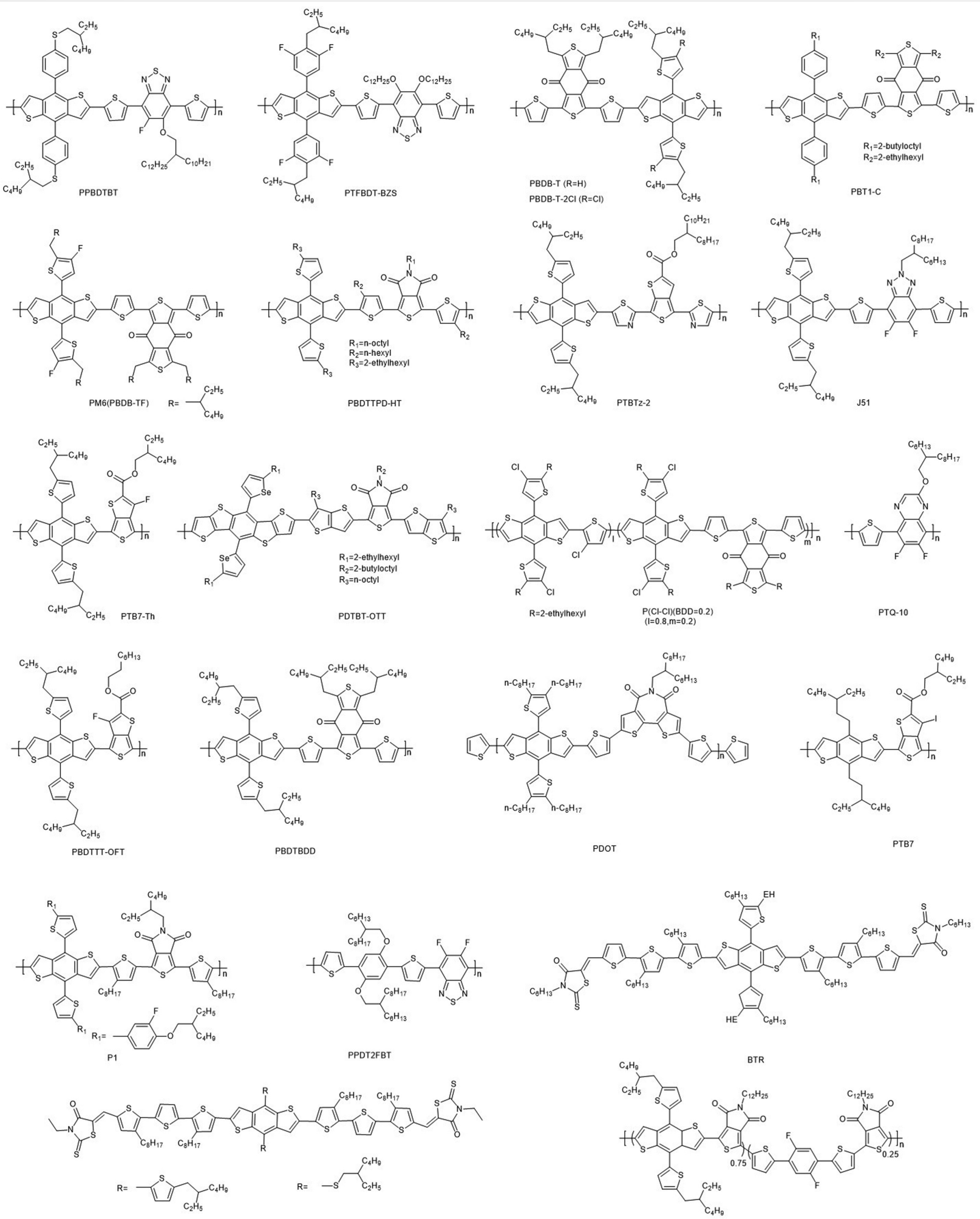

DR3

DR3TSBDT

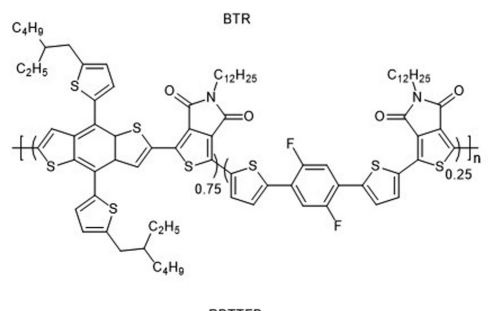

Figure 1 Chemical structures of donor materials. 

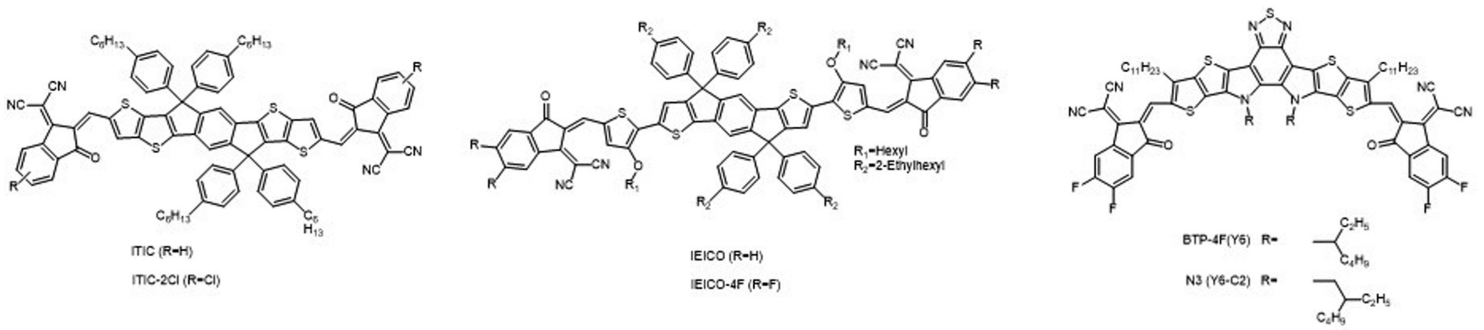

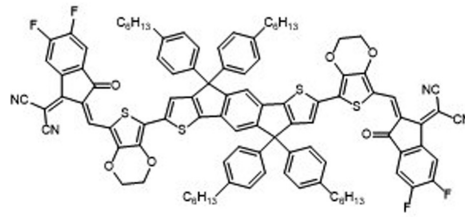

IDT-EDOT

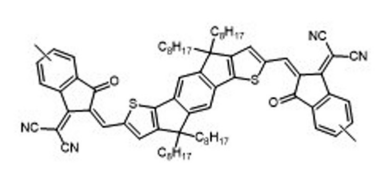

$10 T \cdot 20$

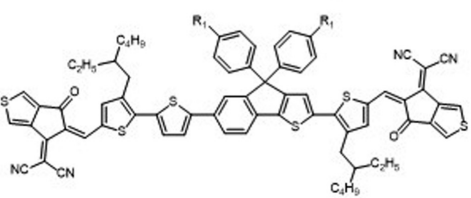

IOTC $\left(\mathbb{R}_{1}-n-\right.$-octy $)$
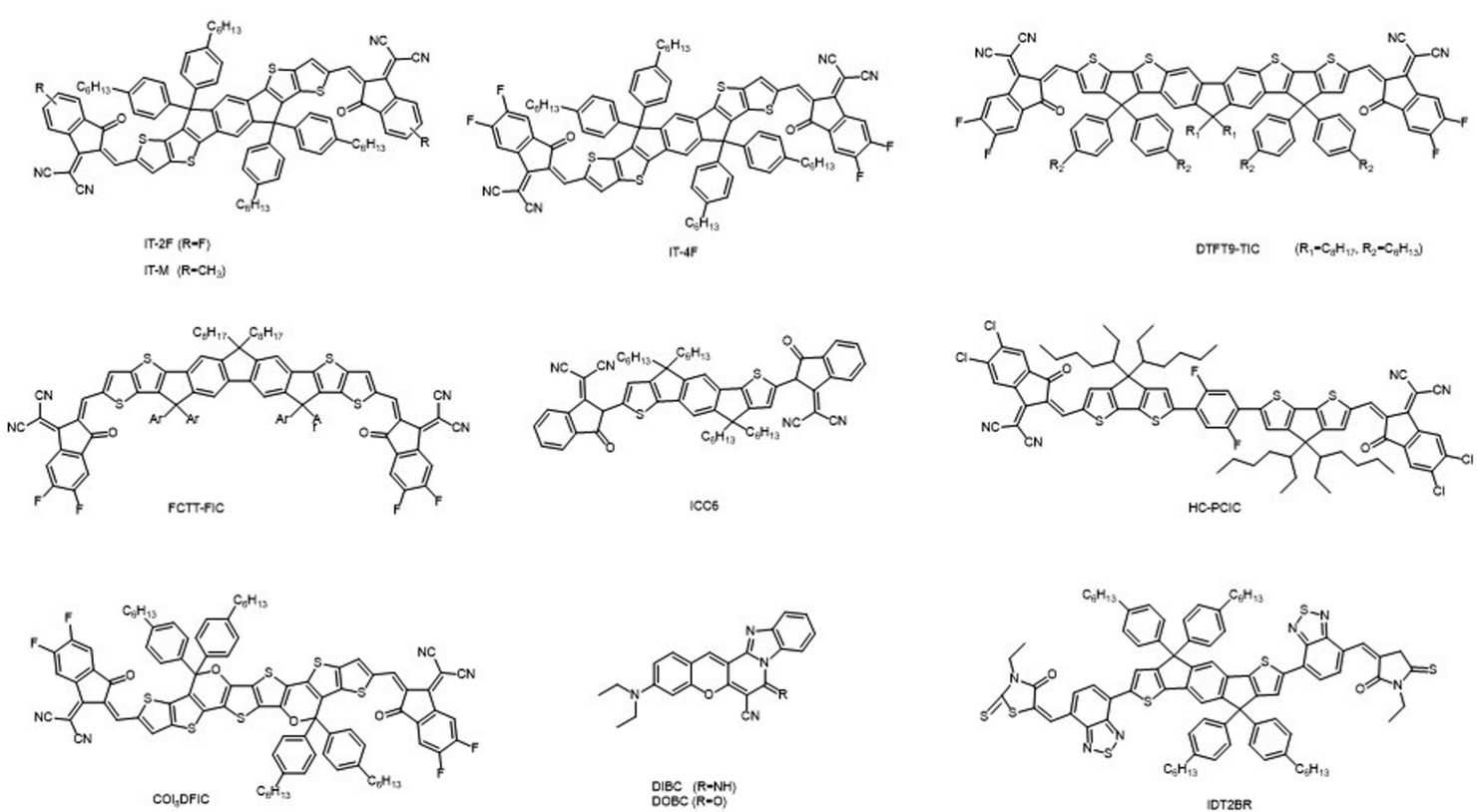

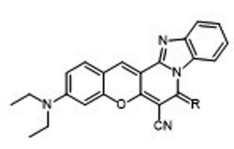

$\mathrm{DIBC}(\mathrm{R}-\mathrm{NH})$
$\mathrm{DOBC}\langle\mathrm{R}=\mathrm{O}\}$

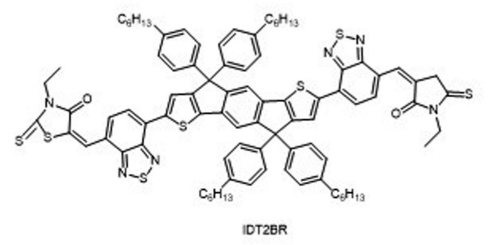

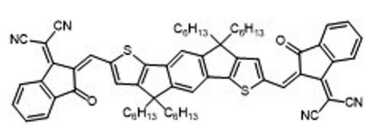

IDIC

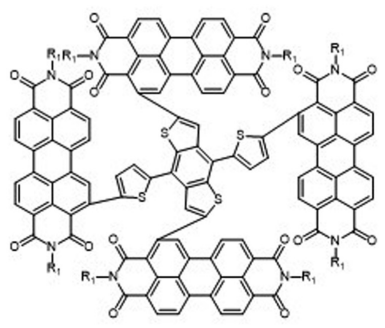

TPB
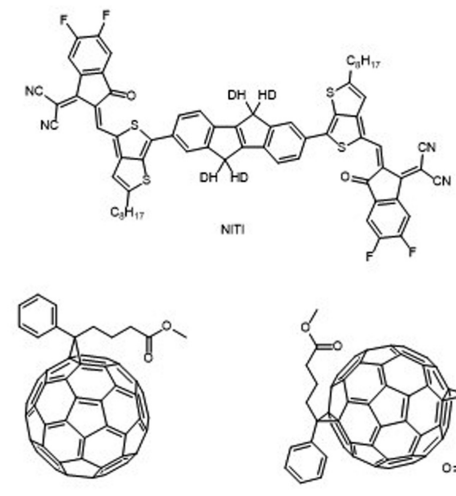

PCEM

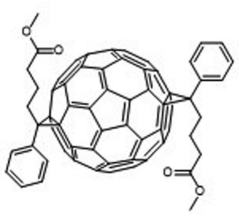

BIS.PCBM
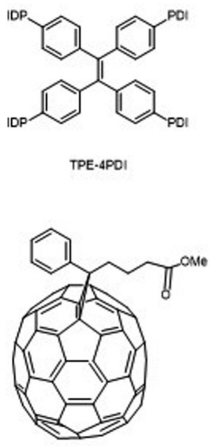

$\mathrm{PC}_{7}, \mathrm{BM}$

Figure 2 Chemical structures of NFAs and fullerene acceptors. 
To settle this matter, Liu et al. introduced the second NFA, leading to a homogeneous mixed phase for superior efficiency. ${ }^{39}$ Zhang et al. reported an IDIC-based solar cell limited by lamellar domains with inerratic crystallite from the aggregation of IDIC. ${ }^{40}$ After mixing an amount of $\mathrm{PC}_{71} \mathrm{BM}$, the lamellar domains disappeared and a new befitting phase separation formed. In other word, the incorporation of FA can disperse the mass aggregation of NFAs and then form a suitable domain size in ternary OSCs to balance charge carrier generation and transport. ${ }^{41-43}$

Based on the ternary strategy involving a FA and an NFA, Lu et al. used 3,9-bis(2-methylene-(3-(1,1-dicyanomethylene)-indanone))-5,5,11,11-tetrakis(4-hexylphenyl)dithieno[2,3-d:2',3'-d']-s-indaceno[1,2-b:5,6-b']dithiophene (ITIC) and $[6,6]$-phenyl- $C_{71}$-butyric acid methyl ester $\left(\mathrm{PC}_{71} \mathrm{BM}\right)$ as acceptors and combined with PPBDTBT (poly \{4,8-bis(4-(2-ethylhexylthio) phen-1-yl)benzo[1,2-b:4,5$b^{\prime}$ ]dithiophen-2,6-yl\}-alt-\{5,5'-(5-(2-decylte-tradecyloxy)6-fluorobenzo[c][1,2,5]thiadiazole-4,7-diyl) di(thiophen-2yl)\}) or PFTBDT-BZS (polymer containing FBDT [4-alkyl-3,5difluorophenyl group was introduced as the lateral side chain to the benzodithiophene (BDT) unit] as the donor unit, benzothiadiazole as the acceptor unit, and thiophene as the spacer) as a donor to fabricate ternary OPVs with the overall D:A ratio of $1: 2$, respectively. Both PPBDTBT: ITIC ${ }^{44}$ and PFTBDT-BZS: ITIC ${ }^{45}$ binary OPVs exhibit limited $J_{\text {sc }}$ of 13.00 and $12.54 \mathrm{~mA} \mathrm{~cm}^{-2}$, as well as the unsatisfied FFs of $63 \%$ and $56 \%$, respectively, which can be attributed to the large phase separation due to the mass aggregation of ITIC, restricting the development of $J_{\mathrm{sc}}$ and FF. Thus, $\mathrm{PC}_{71} \mathrm{BM}$ was added as the third component to disturb the molecular packing of ITIC and reduce its aggregation. As a consequence, a smaller domain size and a larger D/A interface area can be observed in the PPBDTBT: ITIC: PC $_{71} \mathrm{BM}$ ternary blend (with $40 \mathrm{wt} \%$ $\left.\mathrm{PC}_{71} \mathrm{BM}\right)$. By contrast, an inhomogeneous morphology with
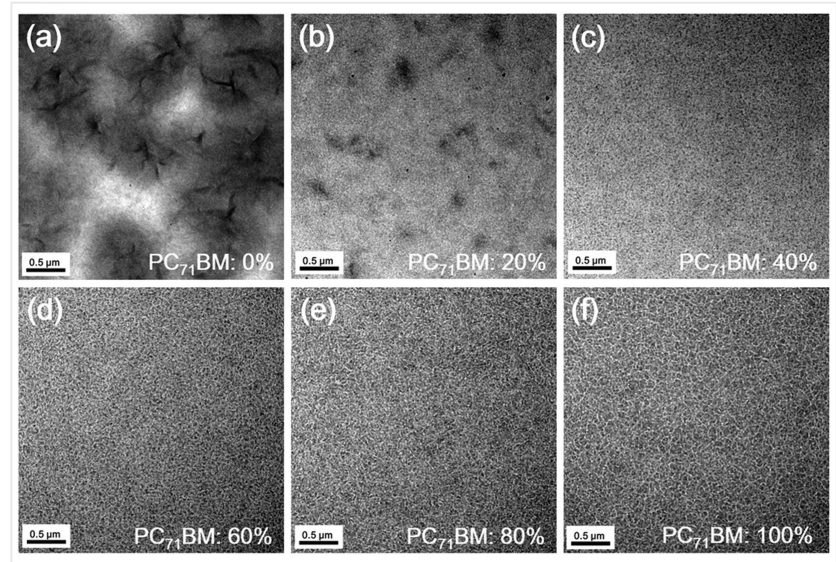

Figure 3 TEM images of PPBDTBT: ITIC: $\mathrm{PC}_{71} \mathrm{BM}$ blend films with different acceptor ratios. Reprinted with permission from Ref. 44. Copyright 2016 Wiley-VCH. a large domain indicating severe aggregation of ITIC in the PPBDTBT: ITIC binary blend was revealed by transmission electron microscopy (TEM) images (Figure 3) and grazingincidence wide-angle X-ray scattering (GIWAXS) diagrams (Figure 4). Therefore, it can be concluded that the
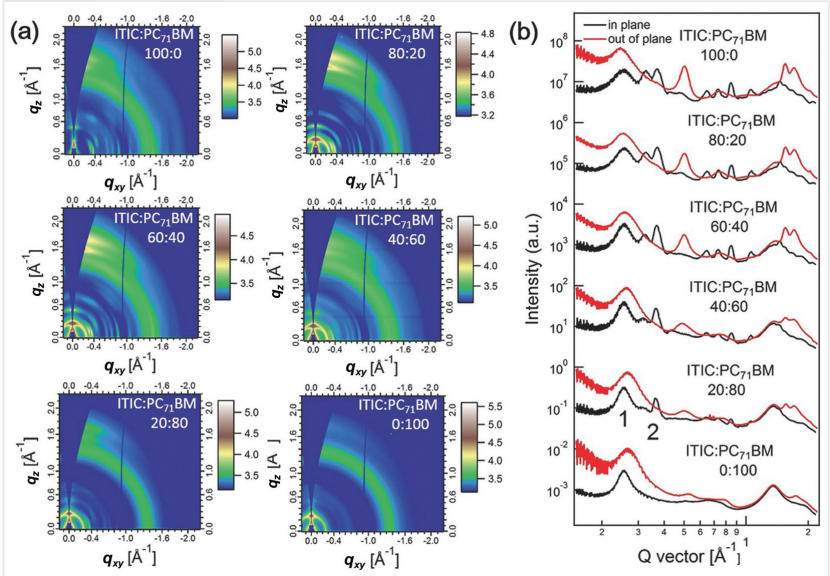

Figure 4 a) GIWAXS 2D scattering patterns and b) line profiles of PPBDTBT: ITIC: PC71BM films with different acceptor ratios. Reprinted with permission from Ref. 44. Copyright 2016 Wiley-VCH.

aggregation of ITIC might be suppressed by $\mathrm{PC}_{71} \mathrm{BM}$. When up to $40 \mathrm{wt} \% \mathrm{PC}_{71} \mathrm{BM}$ was added, the fine phase separation ensured the presence of abundant $\mathrm{D} / \mathrm{A}$ interface areas to boost exciton dissociation. With the increasing content of $\mathrm{PC}_{71} \mathrm{BM}$, nanofibers of the donor material can be observed, which may contribute to $\mathrm{FF}$ improvement by elevated hole transport. Moreover, the ternary blend with the more ordered molecular packing of ITIC and $\mathrm{PC}_{71} \mathrm{BM}$ present enhanced electron mobility, revealed by the results of GIWAXS. With increasing $\mathrm{PC}_{71} \mathrm{BM}$ content, $J_{\mathrm{sc}}$ first increases and then decreases, which might be related to the low absorption coefficient of $\mathrm{PC}_{71} \mathrm{BM}$. When the ratio of ITIC to $\mathrm{PC}_{71} \mathrm{BM}$ was increased up to $1.2: 0.8$, the PPBDTBT: ITIC: PC $_{71}$ BM ternary OPV exhibited a PCE of $10.41 \%$, higher than $7.72 \%$ in the PPBDTBT: ITIC binary OPVs or in ternary OPVs with other ratios of ITIC: $\mathrm{PC}_{71} \mathrm{BM}$, such as PFTBDT-BZS: ITIC: $\mathrm{PC}_{71} \mathrm{BM}$ ternary OPV with the ratio of $1: 0.8: 1.2$.

Zhang et $\mathrm{al}^{46}$ synthesized a fused pentacyclic NFA IDT20 (4,4,9,9-tetraoctyl-4,9-dihydro-s-indaceno[1,2-b:5,6-b'] dithiophene) with strong crystallinity. The ternary OPV consisting of PBDB-T: IDT-20: PC $_{71} \mathrm{BM}$ (1:0.8:0.2) (PBDB-T, poly[(2,6-(4,8-bis(5-(2-ethylhexyl)thiophen-2-yl)-benzo (1,2-b:4,5-b')dithiophene))-alt-(5,5-(1',3'-di-2-thienyl5',7'-bis(2-ethylhexyl)benzo(1', 2'-c:4',5'-c')dithiophene4,8-dione))] achieved a PCE of $10.67 \%$ with a $V_{\text {oc }}$ of $0.87 \mathrm{~V}, \mathrm{a} J_{\mathrm{sc}}$ of $16.80 \mathrm{~mA} \mathrm{~cm}{ }^{-2}$, and a $\mathrm{FF}$ of $72.1 \%$, in contrast to the PBDB-T: IDT-2O (1:1) binary OPV with the PCE of 9.65\%, a $V_{\text {oc }}$ of $0.86 \mathrm{~V}$, a $J_{\mathrm{sc}}$ of $15.70 \mathrm{~mA} \mathrm{~cm} \mathrm{~cm}^{-2}$, and a $\mathrm{FF}$ of $71.6 \%$. Here, the incorporation of FA restrained the 
aggregation of NFA without sacrificing the intense $\pi-\pi$ stacking in the nanophase. The large domains of IDT-20 were seen in the PBDB-T: IDT-20 binary film, but they disappeared after $20 \mathrm{wt} \%$ of $\mathrm{PC}_{71} \mathrm{BM}$ was doped. By contrast, a smoother surface was observed in the optimal ternary blend, revealed by the reduced root-mean-square roughness of $1.70 \mathrm{~nm}$ in the PBDB-T: IDT-20: $\mathrm{PC}_{71} \mathrm{BM}$ ternary blend from $5.47 \mathrm{~nm}$ in the PBDB-T: IDT-20 binary blend. This indicates that $\mathrm{PC}_{71} \mathrm{BM}$ might suppress the aggregation of IDT-2O leading to enlarged D/A interface areas and improved exciton separation. The amorphous active layer experiences a smaller voltage loss with evident aggregation compared with the active layer. ${ }^{47}$ When bulky side chains are introduced to the planar backbone, the aggregation of NFAs would be hampered; however, the $\pi-\pi$ stacking distance between NFA molecules is enlarged, which is unfavorable to electron transport. Besides, because of the excellent electron mobility of the FA and its derivative, such ternary cells, based on a FA and an NFA, display a higher and more balanced hole-electron mobility $\left(\mu_{\mathrm{h}} / \mu_{\mathrm{e}}\right)$, which can facilitate charge transport, as well as reduce trap-assisted recombination, which further promotes $J_{\mathrm{sc}}$. Moreover, Zhang et al. $^{48}$ reported that the PBDB-T: IDT-EDOT: $\mathrm{PC}_{71} \mathrm{BM}$ ternary OPV (IDT-EDOT, a NFA based on $4,4,9,9$ tetrakis(4-hexylphenyl)-4,9-dihydro-s-indaceno[1,2-b:

5,6-b']dithiophene (IDT) and 2-(5,6-difluoro-3-oxo-2,3dihydro-1H-inden-1-ylidene)malononitrile were used as the central donor core and terminal group with 3,4ethylenedioxythiophene as $\pi$-bridging unit) achieved $21 \%$ improvement in PCE compared with the corresponding PBDB-T: IDT-EDOT binary OPV.

To further improve the device efficiency of OPVs, Xu et al. $^{49}$ fabricated an all-small-molecule DR3TSBDT: Y6: $\mathrm{PC}_{71} \mathrm{BM}$ (1.25:0.6:0.4) ternary OPV (DR3TSBDT, 4-thiazolidinone, 5,5'-[[4,8-bis[(2-ethylhexyl)thio]benzo[1,2-b: 4,5-b']dithiophene-2,6-diyl]bis[(3,3"'-dioctyl[2,2':5',2"-terthiophene]-5",5-diyl)methyl-dyne]]bis[3-ethyl-2-thioxo; Y6, (2,2'-((2Z,2'Z)-((12,13-bis(2-ethylhexyl)-3,9-diundecyl12,13-dihydro-[1,2,5] thiadiazolo[3,4-e]thieno[2",3": $\left.4^{\prime}, 5^{\prime}\right]$ thieno[2',3':4,5]pyrrolo[3,2-g]thieno[2',3':4, 5]thieno[3,2b]indole-2,10-diyl)bis(methanylylidene))bis(5,6-difluoro3-oxo-2,3-dihydro-1H-indene-2,1-diylidene))dimalononitrile))bis (5,6-difluoro-3-oxo-2,3-dihydro-1H-indene-2,1diylidene))dimalononitrile) and obtained over 21\% PCE improvement as compared with the corresponding binary OPV based on NFA. First, a smaller phase separation is formed after doping with $40 \mathrm{wt} \% \mathrm{PC}_{71} \mathrm{BM}$ as revealed by the results of TEM. To explore the change in phase separation after doping with a proper amount of FA, Xu et al. employed SasView with the Debye-Anderson-Brumberger model and a fractal model to fit one-dimensional GISAXS profiles and found that the fractal dimension decreased from 3.03 in the binary blend based on Y6 to 2.91 in the ternary blend, suggesting that a looser acceptor packing was formed with an enlarged D/A interface with an improved exciton dissociation. Besides, the good connection between $\mathrm{Y} 6$ and $\mathrm{PC}_{71} \mathrm{BM}$ may benefit to form bi-continuous electron transport channels for the more efficient electron transport. Moreover, the DR3TSBDT molecules in the ternary blend were rearranged and formed a 3D texture structure, which is beneficial for charge transport. As a consequence, the considerably elevated FF (from $55.21 \%$ to $67.27 \%$ ) and the slightly ameliorated $J_{\mathrm{sc}}$ (from 21.67 to $22.19 \mathrm{~mA} \mathrm{~cm}{ }^{-2}$ ) contribute significantly to the increase in device performance (from $10.53 \%$ to $12.84 \%$ ).

Qiu et al. ${ }^{50}$ also adopted $\mathrm{Y} 6$ as the host acceptor, $\mathrm{PC}_{71} \mathrm{BM}$ as the second acceptor, with poly[(thiophene)-alt-(6,7difluoro-2-(2-hexyldecyloxy)quinoxaline)] (PTQ10) as the host donor, and reached a PCE value of $16.07 \%$. The improvement is mainly ascribed to the formation of a favorable face-on orientation packing and the appropriate phase separation, which is responsible for the elevated charge transport and inhibited recombination. Kang et al. ${ }^{51}$ also used $\mathrm{PC}_{71} \mathrm{BM}$ as the second acceptor to dope into the PBDB-T: IOTC binary blend (IOTC, a NFA based on indenothiophene core unit with the side chains of linear 4-n-octylphenyl and 2-(6-oxo-5,6-dihydro-4H-cyclopenta [c] thiophen-4-ylidene)malononitrile (TC) ending group) and achieved improved device performance by decreasing the aggregation of NFA in the binary blend.

In general, a number of research groups have demonstrated that fullerene derivatives can be used as a "morphology regulator" to modify the morphology of NFA-based binary films. ${ }^{43,52-55}$ The addition of FA may properly regulate the phase separation to form a suitable domain. It helps balancing the competition between charge transport and recombination to gain a well-balanced hole/electron mobility and a weak charge recombination, which strongly promotes the values of $J_{\mathrm{sc}}$ and $\mathrm{FF}$. In addition, the incorporation of $F A$, likewise, enlarges the $D / A_{1}$ interface areas and offers an additional $D / A_{2}$ interface or an $A_{1} / A_{2}$ interface for efficient exciton dissociation, in which $A_{1}$ and $\mathrm{A}_{2}$ are the host acceptor and the guest acceptor, and thus improves the device performance.

Crystallinity: The blend crystallinity that affects charge transport can be associated with domain purity. Generally, high crystallinity helps to form bi-continuous interpenetrating networks for efficient charge transport, but might roughen the film surface and may cause insufficient connectivity between the active layer and electrodes. However, low crystallinity might be unfavorable to charge transport. Hence, it is crucial for a well-performed OPV to acquire the desired crystallinity in the active layer; the use of FA as the second acceptor is a promising strategy for finetuning the crystallinity of the active layer in NFA-based binary OPVs.

Hadmojo et al. ${ }^{56}$ investigated how the incorporation of $[6,6]$-phenyl- $C_{70}$-butyric acid methyl ester $\left(\mathrm{PC}_{70} \mathrm{BM}\right)$ into 
the PBDTTPD-HT: ITIC binary OPV (PBDTTPD-HT, a D-A polymer containing benzo[1,2-b:4,5-b'] dithiophene and thieno[3,4-c]pyrrole-4,6-dione groups) affects the crystallinity of the binary blend. The PCE of $12.09 \%$ in the ternary OPV, compared with $9.95 \%$ in the binary-blend OPV, is attributed to the amendatory morphology of the ternary blend. Due to the weak absorption of $\mathrm{PC}_{70} \mathrm{BM}$, the absorption intensity of the ternary OPV was more insufficient than the PBDTTPD-HT: ITIC binary OPV, which corresponds with the higher external quantum efficiency (EQE) value. As can be observed from GIWAXS images (Figure 5), the pristine ITIC film shows a strong orderly

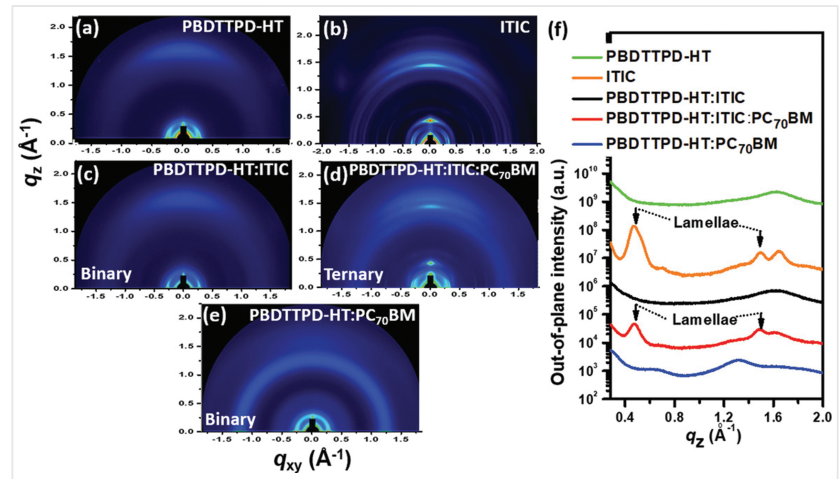

Figure 5 GIWAXS 2D scattering patterns of a) pristine PBDTTPD-HT films, b) pristine ITIC films, c) PBDTTPD-HT: ITIC (1:1.5) binary blend films, d) PBDTTPD-HT: ITIC: PC ${ }_{70} \mathrm{BM}$ (1:0.9:0.6) ternary blend films, and e) PBDTTPD-HT: $P C_{70} B M(1: 1.5)$ binary blend films. f) Out-of-plane line cut of corresponding 2D GIWAXS patterns. Reprinted with permission from Ref. 56. Copyright 2019 Wiley-VCH.

lamellar packing, which, however, nearly disappeared in the binary blend PBDTTPD-HT: ITIC with a weak $\pi-\pi$ stacking diffraction. The characteristic ITIC lamellar ordering reappeared in the ternary blend PBDTTPD-HT: ITIC: $\mathrm{PC}_{70} \mathrm{BM}$, which suggests that ITIC was rearranged after doping with $\mathrm{PC}_{70} \mathrm{BM}$ due to the poorer miscibility between ITIC and $\mathrm{PC}_{70} \mathrm{BM}$ than between ITIC and PBDTTPD-HT. In addition, $\mathrm{PC}_{70} \mathrm{BM}$ has a fine and uniform distribution in the ternary blend, which enlarges the interface between the donor and acceptor domains for efficient exciton dissociation. The continuous frameworks of $\mathrm{PC}_{70} \mathrm{BM}$ and ITIC is beneficial for charge transport. As a result, charge mobility improved without increasing the trap-assistant recombination, leading to boosted charge generation and extraction simultaneously. A higher $J_{\mathrm{sc}}$ of $17.38 \mathrm{~mA} \mathrm{~cm}{ }^{-2}$ and a FF value of $74 \%$ were obtained in the ternary blend OPV, in contrast to a $J_{\mathrm{sc}}$ of $14.86 \mathrm{~mA} \mathrm{~cm}^{-2}$ and a FF of $69 \%$ in the ITIC-based binary OPV.

Fu et al. $^{57}$ used the PBT1-C: IT-2F: PC ${ }_{71}$ BM $(1: 1: 0.2)$ ternary system [PBT1-C, a copolymer with BDT, thiophene, and BDT-4,8-dione (BDTDO) as donor unit, $\pi$ spacer and acceptor unit; IT-2F, 3,9-bis(1-oxo-2-methylene-3-(1,1dicyanomethylene)fluoroindanone)-5,5, 11,11-tetrakis(4n-hexylphenyl)dithieno[2,3d:2',3'd']-s-indaceno[1,2b:5,6b ']dithiophene)] to explore the change in film morphology with the FA as the second acceptor. With $20 \mathrm{wt} \%$ of $\mathrm{PC}_{71} \mathrm{BM}$, the $\pi-\pi$ stacking diffraction peak belonging to IT- $2 F$ decreased in comparison with the PBT1-C: IT-2F binary blend, which means that IT-2F displays lower crystallinity. Atomic force microscopy (AFM) images showed that the thin film based on PBT1-C: IT-2F exhibits many small grains caused by the aggregation of IT-2F, but these disappeared after the introduction of $\mathrm{PC}_{71} \mathrm{BM}$, which means that $\mathrm{PC}_{71} \mathrm{BM}$ can disturb the crystalline aggregates of IT-2F, which agrees well with the abovementioned results. Moreover, $\mathrm{PC}_{71} \mathrm{BM}$ had a fine distribution in the ternary blend and formed an obvious fibrous structure for better charge transport and formed its own domains, causing the reduced recombination, consistent with the increased FF. The incorporation of FAs usually acts as a trap-assisted center for charge recombination, which is, however, suppressed in this ternary system due to the suppressed aggregation of IT2F. Besides, both charge mobility and the probability of charge generation were enhanced in the PBT1-C: IT-2F: $\mathrm{PC}_{71} \mathrm{BM}(1: 1: 0.2)$ ternary OPV, and the ternary OPV achieved an outstanding PCE value of $12.2 \%$, higher than that (11.0\%) of the IT-2F-based binary OPV.

The ternary tactics of using FA as an additional component play a key role in the improvement of high performance of OPV devices. Yan et al. ${ }^{58}$ successfully achieved a record high PCE of $16.67 \%$ using the PM6: Y6: $\mathrm{PC}_{71} \mathrm{BM}$ ternary OPV (PM6 or PBDB-TF, poly[ $(2,6-(4,8$-bis(5(2-ethylhexyl)-4-fluorothiophen-2-yl)benzo[1,2-b:4,5 - $\left.\mathrm{b}^{\prime}\right]$ dithiophene))-co-(1,3-di(5-thiophene-2-yl)-5,7-bis(2-ethylhexyl)-benzo[1,2-c:4,5-c'] dithiophene-4,8-dione))]) at the ratio of $1: 1 \cdot 0: 0.2$. In contrast to the corresponding binary OPV based on PM6: Y6 (1:1.2), the ternary system showed improved $J_{\mathrm{sc}}\left(24.89\right.$ vs. $\left.25.70 \mathrm{~mA} \mathrm{~cm} \mathrm{~cm}^{-2}\right)$ and an improved FF ( $74.37 \%$ vs. $76.35 \%$ ), accounting for the PCE enhancement from $15.75 \%$ to $16.67 \%$. By contrast, the introduction of $\mathrm{PC}_{71} \mathrm{BM}$ makes up for the weak absorption of the binary blend PM6: Y6 OPVs in short wavelength. On the other hand, the morphology of thin film is modified after the proper content of $\mathrm{PC}_{71} \mathrm{BM}$ is doped. More $\mathrm{D} / \mathrm{A}$ interface pathways in ternary blends were formed to accelerate charge extraction, which is shown by the high charge collection probability $(P(E, T))$ of 0.970 as compared with 0.955 in the Y6-based binary blend. From GIWAXS images (Figure 6), it was found that as doping with the isotropic $\mathrm{PC}_{71} \mathrm{BM}$, the "face-on" preferred orientation is withheld in the ternary blend for favorable intermolecular charge transport, and a similar phenomenon was also observed by Dai et al., ${ }^{59}$ resulting in a high and balanced hole/electron mobility as well as a suppressed charge recombination. Moreover, the coherent length related to $\pi-\pi$ stacking in the 


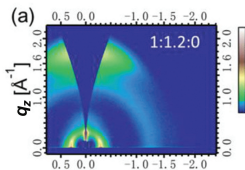

$q_{x y}\left[\AA^{-1}\right]$
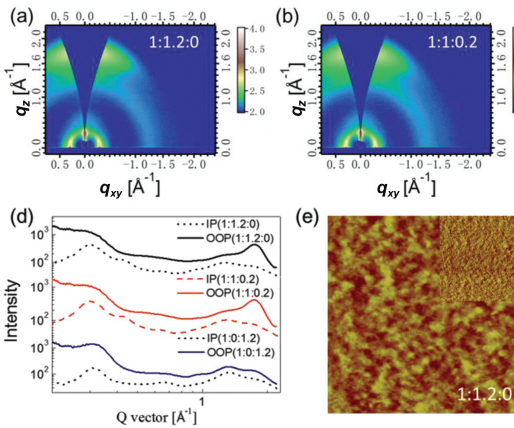

$q_{x y}\left[\AA^{-1}\right]$
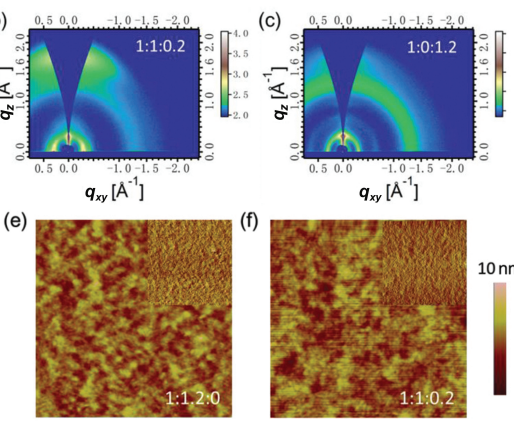

$q_{x y}\left[A^{-1}\right]$

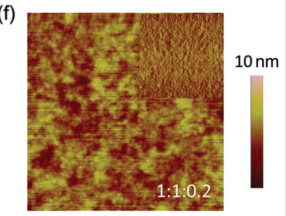

Figure 6 GIWAXS 2D scattering patterns of PM6: Y6: $P C_{71} B M$ films with different ratios a) 1:1.2:0; b) 1:1:0.2; and c) 1:0:1.2. d) Out-of-plane (solid line) line and in-plane (dotted line) line cut of corresponding 2D GIWAXS patterns; and AFM images $(2 \mu \mathrm{m} \times 2 \mu \mathrm{m})$ of films with blend ratio of e) $1: 1.2: 0$ and f) $1: 1: 0.2$. Reprinted with permission from Ref. 58. Copyright 2019 Wiley-VCH.

out-of-plane direction increased from 1.628 to $1.954 \mathrm{~nm}$ with the presence of $\mathrm{PC}_{71} \mathrm{BM}$, corresponding to the higher crystallinity of the ternary blend for better charge generation and transport. In other reports, the higher crystallinity with the lengthened coherent lengths or the decreased $\pi-\pi$ stacking distance would be beneficial to the formation of a bi-continuous interpenetrating network, as well as to further improve charge transport and collection and reduce recombination. ${ }^{60,61}$ Yan et al. also verified that there is an energy transfer between $\mathrm{Y} 6$ and $\mathrm{PC}_{71} \mathrm{BM}$ when the third component is over $30 \%$. Once the content of the third component exceeds $30 \%$, the effect of a parallel mechanism should also be considered. This phenomenon suggests that the working mechanism of ternary OPVs would change with the diversification of the blending ratio.

Fullerene derivatives were used as the third component to modify thin-film morphology towards a more suitable blend crystallinity, leading to a fibrous or continuous interpenetrating network to provide more charge transport channels for efficient charge transport. ${ }^{62-66}$ It might be possible to modify NFA-based binary OPVs with insufficient charge mobility caused by poor morphology. ${ }^{67}$ Thus, more balanced charge mobility with increased electron mobility is formed. All of this can provide a reasonable explanation for improved PCEs with higher $J_{\text {sc }}$ values in the ternary devices compared to NFA-based binary devices. These findings showed that FAs could modulate the crystallinity of the active layer involved with NFAs to facilitate charge transport, extraction, and restrain charge recombination, which is beneficial for improving the device performance.

Vertical distribution: There is a report that has found that in conventional OSCs, most excitons are generated at the bottom of the conventional OSCs, and thus the electron needs to transport a long distance toward the cathode, ${ }^{68}$ which indicates that the electron transport property strongly affects the device performance. The introduction of a FA into a donor: an NFA blend can help the formation of an ideal vertical distribution ${ }^{69-71}$; donor and acceptor may enrich in the active layer close to the anode and cathode, respectively, which would shorten the distance of charge transport and enhance charge collection, further improving $J_{\text {sc }}$ and FF.

Bi et al. used a spin-coated PBDB-T: ITIC: $\mathrm{PC}_{71} \mathrm{BM}(30$ wt\%) ternary OPV to study the effect of the second acceptor, FA, on the vertical phase distribution of both donor and acceptor. $^{72}$ The film-depth-dependent light absorption spectrum results for the ternary blend and the corresponding binary blends are shown in Figure 7a-c. The peaks at 580 and $620 \mathrm{~nm}$ are from PBDB-T, and the peak centered at
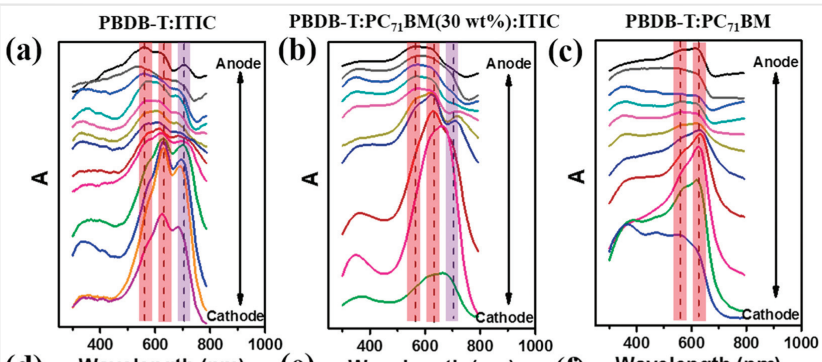

(d)
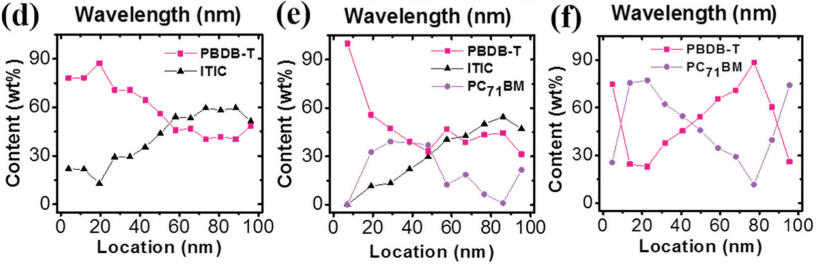

Figure 7 Film-depth-dependent light absorption spectra of PBDB-T: ITIC: $P C_{71} B M$ films with different $P C_{71} B M$ contents: a) $0 \%$; b) $30 \%$; and c) $100 \%$. (d-f) PBDB-T, ITIC, and $\mathrm{PC}_{71} \mathrm{BM}$ content profiles at different film depths by fitting the evolution of spectroscopy as shown in (a-c). (In composition profiles, locations at 0 and $100 \mathrm{~nm}$ are active layer- $\mathrm{MoO}_{3}$ (anode) and active layer-ZnO (cathode) interfaces.) Reprinted with permission from Ref. 72. Copyright 2018 Elsevier.

$700 \mathrm{~nm}$ is arising from ITIC; the spectra of the three different materials varied with the film depth. It is obvious that the ternary blend displayed ideal vertical phase separation (Figure $7 d-f$ ), which is mainly rich in PBDB-T and ITIC in the active layer close to the anode and cathode respectively, which is beneficial for accelerating charge collection as well as reducing recombination. In addition, the increased hole and electron mobility owes to the outstanding charge transport property of $\mathrm{PC}_{71} \mathrm{BM}$ and the desired coherence length of PBDB-T, as well as ITIC. The smaller aggregation of the polymer with an enlarged $D / A$ interface area for efficient exciton dissociation after mixing with $\mathrm{PC}_{71} \mathrm{BM}$ can be observed from the time-resolved fluorescence images. The ternary cell with $30 \mathrm{wt} \% \mathrm{PC}_{71} \mathrm{BM}$ reached $11.0 \%$ PCE, taking advantage of enhanced $J_{\mathrm{sc}}$ and FF in contrast to $9.6 \%$ PCE of the binary cell based on NFA. 
In a research study of the invented ternary device based on PTB7-Th: IEICO-4F: PC ${ }_{71}$ BM (PTB7-Th, poly[[2,6'-4,8-di(5ethylhexyl-thienyl)benzo[1,2-b;3,3-b]dithiophene][3-fluoro-2[(2-ethylhexyl)carbonyl]thieno[3,4-b]-thiophenediyl]]; IEICO-4F, 2,2'-((2Z, 2'Z)-(((4,4,9,9-tetrakis(4-hexylphenyl)4,9-dihydro-s-indaceno [1,2-b:5,6-b']di-thiophene-2,7-diyl) bis(4-((2-ethylhexyl)-oxy) thiophene-5,2-diyl))bis(methanylylidene))bis(5,6-difluoro-3-oxo-2,3-dihydro-1H-indene-

2,1-diylidene))dimalononitrile), Lee et al. found that the vertical distribution might be related to the surface energy of different materials. ${ }^{73}$ In view of the relatively high surface energy of $\mathrm{PC}_{71} \mathrm{BM}$ and the high surface energy of $\mathrm{ZnO}$, the former tends to aggregate in the surface of the latter during film formation, which helps to effectively inhibit charge recombination, boost charge collection, and block hole leakage. However, PTB7-Th was enriched near the BHJ/air interface because of the low surface energy, and thus the desired vertical distribution was formed. Because of the similar surface energy between $\mathrm{PC}_{71} \mathrm{BM}$ and IEICO-4F, the latter was also enriched at the $\mathrm{ZnO}$ surface, and IEICO-4F molecules could interact closely with $\mathrm{PC}_{71} \mathrm{BM}$ molecules, which may enhance device performance. As a result, the ternary OPV exhibits improved PCE (from 9.44\% to $10.55 \%$ ) with the higher value of $J_{\mathrm{sc}}$ (from 21.30 to $22.64 \mathrm{mAcm}^{-2}$ ) and showed superior stability (maintaining $80 \%$ of PCE under continuous illumination for $500 \mathrm{~h}$ ). This indicates that the ternary strategy can improve device performance and stability simultaneously, and thus achieve the commercialization of OPVs.

Park et al. ${ }^{74}$ introduced $\mathrm{PC}_{71} \mathrm{BM}$ as the guest acceptor into the $\mathrm{P}(\mathrm{Cl}-\mathrm{Cl})(\mathrm{BDD}=0.2)$ : IT- $4 \mathrm{~F}$ binary blend to fabricate an inverted ternary device $(\mathrm{P}(\mathrm{Cl}-\mathrm{Cl})(\mathrm{BDD}=0.2)$, a polymer consisting of a chlorinated $2 \mathrm{D}$ benzo(1,2-b:4,5- $\left.\mathrm{b}^{\prime}\right)$ dithiophene (Cl-2DBDT) donor unit, a 3-chlorothiophene (Cl-Th) acceptor unit, and a 1,3-bis(5-bromothiophen-2-yl)5,7-bis(2-ethylhexyl)benzo(1,2-c:4, 5-c')dithiophene-4,8dione (BDD) acceptor unit with the ratio of $\mathrm{Cl}-2 \mathrm{DBDT}$ : $\mathrm{Cl}-$ Th: BDD = 1:0.8:0.2; IT-4F, (3,9-bis(1-oxo-2-methylene-3(1,1-dicyanomethylene)-5,6-difluoroindanone)-5,5, 11,11tetrakis(4-n-hexylphenyl)dithieno[2,3d:2',3'd']-s-indaceno $\left[1,2 b: 5,6 b^{\prime}\right]$ dithiophene)), and surprisingly noted that the formation of favorable vertical phase separation can decrease the interface resistance and recombination. Park et al. used the cross-sectional field-emission scanning electron microscopy and energy-dispersive X-ray spectroscopy to analyze the vertical distribution of three different materials in the film via various atomic signals of $F_{1 s}, S_{2 p}$, $\mathrm{O}_{1 \mathrm{~s}}$, and $\mathrm{C}_{1 \mathrm{~s}}$, in which the $\mathrm{S}_{2 \mathrm{p}}$ signal represents $\mathrm{P}(\mathrm{Cl}-\mathrm{Cl})$ $(B D D=0.2)$, the $F_{1 s}$ signal stands for IT- $4 F$, and the $O_{1 s}$ and $\mathrm{C}_{1 \mathrm{~s}}$ signals represent $\mathrm{PC}_{71} \mathrm{BM}$. After loading $10 \mathrm{wt} \% \mathrm{PC}_{71} \mathrm{BM}$, the $\mathrm{F}_{1 \mathrm{~s}}$ signals showed that $\mathrm{P}(\mathrm{Cl}-\mathrm{Cl})(\mathrm{BDD}=0.2)$ mainly appeared in the upper portion of the active layer, while the $\mathrm{S}_{2 \mathrm{p}}$ signals representing IT- $4 \mathrm{~F}$ as well as the $\mathrm{O}_{1 \mathrm{~s}}$ and $\mathrm{C}_{1 \mathrm{~s}}$ signals representing $\mathrm{PC}_{71} \mathrm{BM}$ gathered in the nether portion of the active layer, in accordance with the well-dispersed vertical phase distribution. Subsequently, a uniform morphology and fine phase separation could be observed in the AFM images of the ternary blend. The effective contact between the active layer and electrode reduced the value of interface resistance, from $105.01 \Omega$ in the binary device to $74.83 \Omega$ in the ternary device; furthermore, the value of $\mathrm{EQE}$ in the region of $550-800 \mathrm{~nm}$ is over $80 \%$, indicating the enhanced charge transport and extraction in ternary device, and it would be reasonable to infer that the ternary strategy can reduce interface resistance and boost charge carrier transport and collection via forming a vertical phase separation. As a consequence, $J_{\mathrm{sc}}$ of the ternary OPV increased from $20.00 \mathrm{~mA} \mathrm{~cm}^{-2}$ in the binary OPV based on IT-4F to $21.2 \mathrm{~mA} \mathrm{~cm}^{-2}$.

To conclude, the incorporation of FA is favorable to the formation of an ideal vertical phase separation, which may be related to the surface energy of the different materials, leading to an improvement in performance with increased charge carrier transport, collection, and suppressed recombination.

\section{Mending Charge Dynamics}

The charge dynamics play a significant role in device performance and it restricts further performance improvement of NFA-based binary OPVs. The incorporation of FA into binary OPVs based on NFA can improve the electron mobility due to the outperformed electron mobility of FAs, further optimizing $\mu_{\mathrm{h}} / \mu_{\mathrm{e}}$ and reducing recombination, consistent with a high FF. ${ }^{75-86}$ Besides that, the exciton dissociation is also enhanced, to boost the photoinduced current via the enlarged $\mathrm{D} / \mathrm{A}$ interface. The wide application of the ternary strategy in many different binary systems based on NFA can support that the use of FA as the third component can improve device efficiency. This indicates that the ternary strategy is promising.

Shi et al.'s ${ }^{87}$ research attested that the charge mobility dynamics strongly affect cell efficiency. The binary solar cell based on the J52: IEICO-4F binary solar cell (J52, poly\{4,8bis[5-(2-ethylhexyl)thiophen-2-yl] benzo[1,2-b:4,5-b'] dithiophene-5,5'-diyl-alt-4,7-bis(thien-2-yl)5,6-difluoro2-(2-hexyldecyl)-2H-benzo[d][1,2,3]triazole\}) shows hole and electron mobilities of $1.42 \times 10^{-3}$ and $9.36 \times 10^{-5} \mathrm{~cm}^{2}$ $\mathrm{V}^{-1} \mathrm{~s}^{-1}$, respectively. The enormously unbalanced $\mu_{\mathrm{h}} / \mu_{\mathrm{e}}$ ratio causes a low $\mathrm{FF}$, thus confining device efficiency. After adding $40 \mathrm{wt} \% \mathrm{PC}_{71} \mathrm{BM}$ to the binary solar cell, the electron mobility was improved up to $9.35 \times 10^{-4} \mathrm{~cm}^{2} \mathrm{~V}^{-1} \mathrm{~s}^{-1}, 1$ order of magnitude higher than that of the binary cell. The $\mu_{\mathrm{h}} / \mu_{\mathrm{e}}$ ratio decreases from 15 to 1.5 for an improved FF. Furthermore, the suppression of recombination, together with elevated charge extraction and transport, converged to achieve PCEs of $10.68 \%$ as compared with $9.21 \%$ for binary OPV based on J52: IEICO-4F. 
To investigate the issue of insufficient charge separation in NFA-based binary OPVs, Wang et al. ${ }^{88}$ fabricated a binary OPV, using PTB7-Th as a host donor and IEICO-4F as a host acceptor, and further used $\mathrm{PC}_{71} \mathrm{BM}$ as a guest acceptor. In the PTB7-Th: IEICO-4F binary blend, PTB7-Th did mix well with IEICO- $4 \mathrm{~F}$ because of the excellent miscibility between them, which, however, leads to severe charge carrier recombination, corresponding to the short lifetimes of the free charge carriers. In ternary OPVs, $\mathrm{PC}_{71} \mathrm{BM}$ may reduce the chargetransfer states and thus facilitate the interfacial charge transfer. As a result, $J_{\mathrm{sc}}$ increased to $23.63 \mathrm{~mA} \mathrm{~cm}^{-2}$ in the ternary device from $20.62 \mathrm{~mA} \mathrm{~cm}{ }^{-2}$ in the binary device based on IEICO-4F.

To better explore the effects of exciton dissociation and charge transport on the photovoltaic performance, Li et al. ${ }^{89}$ used pump-probe photoconductivity spectroscopy measurements combined with transient photoluminescence (PL) spectroscopy. The transient PL measurement can monitor the charge transfer by using a selected wavelength to excite one component and to probe its PL emission intensity. After blending with the other component (cannot be excited in this wavelength), the shorter PL intensity lifetime indicates that effective charge transfer occurs ${ }^{67,90}$ but the longer PL intensity lifetime may mean the existence of energy transfer. ${ }^{58}$ In addition to transient PL measurement, the transient absorption (TA) spectroscopy can also investigate charge transfer and combination dynamics by monitoring ground-state bleaching and photo-induced polarons. ${ }^{72,91-93}$ As for the PBDB-T: ITIC binary OPV, it is found that the excitons generated in ITIC would separate at the PBDB-T/ITIC interface, then the electrons and holes would transfer to the cathode and the PBDB-T, respectively. The accumulation of the injected holes plus the holes generated in PBDB-T would inhibit the exciton generation in PBDB-T. Fortunately, the addition of $\mathrm{PC}_{71} \mathrm{BM}$ can enlarge the $\mathrm{D} / \mathrm{A}$ interface area. Although the exciton dissociation at the ITIC/PC ${ }_{71} \mathrm{BM}$ interface can be ignored, that in PBDB-T/PC ${ }_{71} \mathrm{BM}$ is efficient. Besides that, the $\mathrm{PC}_{71} \mathrm{BM}$ would be able to accept electrons from PBDB-T, and the electron transfer to the cathode is faster than that in ITIC; thus, the ternary device exhibits a larger hole mobility $\left(1.94 \times 10^{-4}\right.$ vs. $1.01 \times 10^{-4} \mathrm{~cm}^{2} \mathrm{~V}^{-1} \mathrm{~s}^{-1}$ ) and a lower hole-trapping state than the PBDB-T: ITIC binary device $\left(3.25 \times 10^{16}\right.$ vs. $4.15 \times 10^{16} \mathrm{~cm}^{-3}$ ) with reduced hole accumulation, which is favorable for charge separation and transport, consistent with the improved $J_{\mathrm{sc}}$ in ternary OPV. As a result, the ternary OPV achieved higher PCEs (10.43\% vs. 9.03\% in PBDB-T: ITIC binary OPV) depending on the increased probability of exciton dissociation and charge mobility.

Cheng et al. ${ }^{94}$ synthesized an extremely planar NFA, incorporating two carbon bridges to fasten the central DTF core with two outer thiophene rings, forming a new laddertype nonacyclic dithieno[3,2-b:6,7-b'] fluorenedi(cyclopentathiophene) (denoted as DTFT9), which combined with a polymer donor, PBDB-T, resulting in an efficiency of $9.58 \%$. And DTFT9 was further condensed with two FIC acceptor units (FIC, 1,1-dicyanomethylene-5,6-diuoro-3-indanone) to yield a carbon-bridged DTFT9-FIC. The PBDB-T: DTFT9FIC binary OPV exhibits a "face-on" orientation, while the ternary OPV PBDB-T: DTFT9-FIC: PC ${ }_{71}$ BM shows "face-on" and "edge-on" orientations, which may favor charge transport in the vertical and parallel directions. In other words, the charge mobility of PBDB-T: DTFT9-FIC: $\mathrm{PC}_{71} \mathrm{BM}$ OPV is superior to that of the PBDB-T: DTFT9-FIC binary $\mathrm{OPV}$, and the former is more balanced, which is redound to elevate the value of FF. Furthermore, the PBDB-T: DTFT9FIC: $\mathrm{PC}_{71} \mathrm{BM}$ ternary OPV shows a smoother surface with smaller roughness than the binary OPV based on PBDB-T: DTFT9-FIC, which indicates a better miscibility in the ternary blend, enhancing the interface contact and boosting charge transport. The ternary OPV achieved an efficiency of $11.82 \%$ with increased $J_{\mathrm{sc}}$ and $\mathrm{FF}$ values. The same group used $\mathrm{PC}_{71} \mathrm{BM}$ as the second acceptor to combine with the PM6: FCTT-FIC binary blend (FCTT-FIC, two outer thienothiophenes-terminal fluorenedicyclopentathieno[3,2-b] thiophene condensed with two FIC acceptor units and a carbon bridge), and achieved a PCE of $13.37 \%$ in the ternary OPV, in contrast to a PCE of $12.23 \%$ in the binary OPV of PM6: FCTT-FIC. ${ }^{95}$ The improvement in device performance is mainly ascribed to the increased FF, which stems from the increased mobility. The electron mobility increased from $2.50 \times 10^{-5} \mathrm{~cm}^{2} \mathrm{~V}^{-1} \mathrm{~s}^{-1}$ in the binary device based on PM6: FCTT-FIC to $3.03 \times 10^{-5} \mathrm{~cm}^{2} \mathrm{~V}^{-1} \mathrm{~s}^{-1}$ in the ternary device, and the hole mobility raised from $4.71 \times 10^{-6} \mathrm{~cm}^{2} \mathrm{~V}^{-1} \mathrm{~s}^{-1}$ in the binary device based on PM6: FCTT-FIC to $2.32 \times 10^{-5} \mathrm{~cm}^{2} \mathrm{~V}^{-1} \mathrm{~s}^{-1}$ in the ternary device, leading to the higher and more balanced charge transport and thus a higher PCE with enhanced FF and $J_{s c}$ values.

Luo et al. designed and synthesized a series of smallmolecule NFA acceptors based on Y6, Y6-C2 ((2,2'-((2Z,2-Z)((12,13-bis(3-ethylheptyl)-3,9-diundecyl-12,13-dihydro$[1,2,5]$ thiadiazolo[3,4-e]thieno[2",3": $\left.4^{\prime}, 5^{\prime}\right]$ thieno[2',3':4,5] pyrrolo[3,2-g]thieno[ $\left.2^{\prime}, 3^{\prime}: 4,5\right]$ thieno[3,2-b]indole-2,10diyl)bis(methaneyl-ylidene))bis(5,6-difluoro-3-oxo-2,3dihydro-1H-indene-2,1-diy-lidene))dimalononitrile), by altering the branching position of the side alkyl chains. ${ }^{96}$ The PM6: Y6-C2 binary cell achieved a high performance of 15.89\%. To further increase device performance, they incorporated an amount of $\mathrm{PC}_{71} \mathrm{BM}$ into the PM6: Y6-C2 binary cell and achieved a higher PCE of $17.06 \%$, which is mainly ascribed to the higher mobility and the more balanced $\mu_{\mathrm{h}} / \mu_{\mathrm{e}}$ ratio. It is worth noting that the improved charge transport features could reduce recombination to some degree, accounting for the slightly increased $J_{\mathrm{sc}}$ and the strongly improved $\mathrm{FF}$, from $73.6 \%$ to $77.2 \%$.

Hou et al. mixed an appropriate amount of [6,6]-phenyl$\mathrm{C}_{61}$-butyric acid methyl ester $\left(\mathrm{PC}_{61} \mathrm{BM}\right)$ into the PBDB-TF: $\mathrm{Y} 6$ binary OPV and achieved a high PCE of $16.5 \% .{ }^{97}$ Because the 
LUMO energy level is higher for $\mathrm{PC}_{61} \mathrm{BM}$ than for $\mathrm{Y} 6$, the $V_{\mathrm{oc}}$ values slightly improved in the ternary cell. The electron mobility improves with the increasing $\mathrm{PC}_{61} \mathrm{BM}$ contents, whereas the hole mobility is insensitive to the loading of $\mathrm{PC}_{61} \mathrm{BM}$ (Figure 8). When the ratio of $\mathrm{Y} 6: \mathrm{PC}_{61} \mathrm{BM}$ increased up to 1:0.2, the charge carrier mobility of the PBDB-TF: Y6:
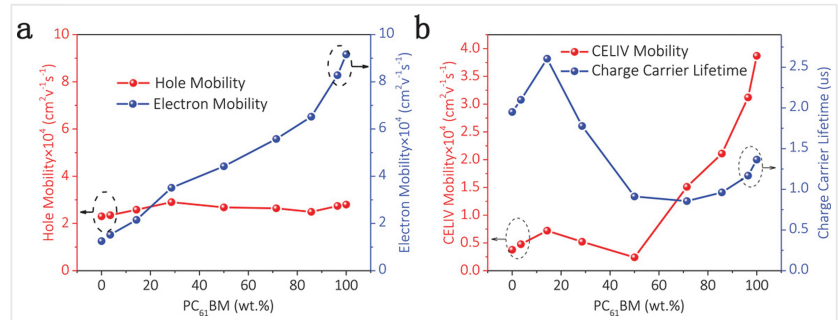

Figure 8 a) The hole and electron mobilities and b) the charge carrier lifetimes and CELIV mobilities of the ternary blends with different $P C_{71} B M$ content (wt\%) in $A_{1}: A_{2}$. Reprinted with permission from Ref. 97. Copyright 2019 Wiley-VCH.

$\mathrm{PC}_{61} \mathrm{BM}(1: 1.2: 0.2)$ ternary blend was $7.3 \times 10^{-5} \mathrm{~cm}^{2} \mathrm{~V}^{-1}$ $\mathrm{s}^{-1}$, an increase of nearly a factor of 2 over the PBDB-TF: Y6 blend $\left(3.8 \times 10^{-5} \mathrm{~cm}^{2} \mathrm{~V}^{-1} \mathrm{~s}^{-1}\right)$. With further increase in $\mathrm{PC}_{61} \mathrm{BM}$ content, the charge carrier mobility decreases slightly, which can be correlated with the variation in blend morphology. The PBDB-TF: Y6: $\mathrm{PC}_{61} \mathrm{BM}(1: 1.2: 0.2)$ ternary OPV displays the highest charge carrier mobilities and the longest charge carrier lifetimes among PBDB-TF: Y6: $\mathrm{PC}_{61} \mathrm{BM}$ ternary OPVs with other ratios (from 1:1.4:0 to $1: 0: 1.4)$, which restrains charge recombination and accounts for increased $\mathrm{FF}$ and $J_{\mathrm{sc}}$. Hou et al. also combined $\mathrm{PC}_{61} \mathrm{BM}$ with PBDB-T-2Cl: BTP-4F (PBDB-T-2Cl, a polymer based on PBDB-T with chlorinated thiophene side groups; BTP-4F, also known as Y6), and gained a higher and more balanced charge mobility, leading to a PCE of $16.3 \% .^{98}$ The PBDB-T-2Cl: BTP-4F: PC $_{61}$ BM ternary OPV with a $300 \mathrm{~nm}$ active layer reached a PCE of $14.3 \%$, which is important for roll-to-roll production.

The abovementioned discussion indicates that introducing FA as the second acceptor into NFA-based binary OPVs may achieve the more balanced and higher charge mobility, further leading to higher PCEs with improved $J_{\mathrm{sc}}$ and FF values.

\section{Other Effects}

Apart from modifying morphology and improving charge dynamics, the incorporation of FA into binary OPVs based on NFA can increase PCE by virtue of other effects such as energy transfer, cascade energy level alignment, $^{99-102}$ and others. ${ }^{103,104}$
Energy transfer: Fullerene derivatives as the third component with absorption in the short wavelength region usually serve as an energy donor to form energy transfer to the NFAs. Steady and transient PL measurements are an efficient method of verifying the existence of energy transfer. If the PL emission intensity of a low-bandgap acceptor (energy acceptor) increased and the PL emission intensity of a large-bandgap acceptor (energy donor) decreased after intermixing two acceptors, it means that an energy transfer occurred between them. Karuthedath et al. ${ }^{91}$ incorporated $\mathrm{PC}_{71} \mathrm{BM}$ into the binary OPV based on DR3: ICC6 (DR3, oligothiophenes containing a backbone structure of a BDT unit as the central block and ethylrhodanine as the end group) to improve overall device performance by the ultrafast energy transfer between $\mathrm{PC}_{71} \mathrm{BM}$ and ICC6. $\mathrm{PC}_{71} \mathrm{BM}$ absorbs additional energy from high-energy photons, then quickly transfers it to ICC6, and subsequently ICC6 transfers holes to DR3. Furthermore, due to the inherent good electron transport property of $\mathrm{PC}_{71} \mathrm{BM}$, the ternary OPV with $\mathrm{PC}_{71} \mathrm{BM}$ exhibits an increased $\mathrm{EQE}$ in the whole absorption range and increased charge extraction as well as reduced recombination. ${ }^{105}$ As a result, the ternary OPV provides improved $J_{\mathrm{sc}}$ (from 15.2 to $16.3 \mathrm{~mA} \mathrm{~cm}^{-2}$ ) and FF values (from $64 \%$ to $72 \%$ ), which contributes to an increased PCE of $10.8 \%$ compared with $8.7 \%$ in the DR3: ICC6 binary OPV.

Cascade energy alignment: The addition of a FA into a binary system based on an NFA may help to form cascade energy alignment. Zhan et al. ${ }^{106}$ introduced $\mathrm{PC}_{71} \mathrm{BM}$ as the second acceptor into PBDB-TF: HC-PCIC binary systems (HC-PCIC, 2,2'-((2Z,2'Z)-(((2,5-difluoro-1,4-phenylene)bis (4,4-bis(2-ethylhexy -1)-4H-cyclopenta[2,1-b:3,4-b']dithiophene-6,2-diyl))bis(methan-ylylidene))bis(5,6-dichloro-3oxo-2,3-dihydro-1H-indene-2,1-diylidene))dimalononitrile) and achieved a higher PCE (12.36\%) with a $J_{\text {sc }}$ of $19.29 \mathrm{~mA} \mathrm{~cm}^{-2}$ compared with a PCE of $11.48 \%$ with a $J_{\mathrm{sc}}$ of $17.54 \mathrm{~mA} \mathrm{~cm}^{-2}$ in HC-PCIC-based binary systems. The addition of $\mathrm{PC}_{71} \mathrm{BM}$ formed a cascade energy level alignment, leading to enhanced charge transfer between the donor and the acceptor, as proved by the increased quantum efficiencies in the range of $600-800 \mathrm{~nm}$. In addition, the enhanced charge transfer would make a positive difference in charge separation, revealed by the PL spectrum. The pure PBDB-TF displayed a PL spectrum in the range of 625-850 nm. After doping with HC-PCIC, most of the fluorescence emission is quenched, but some of it still remains in the range of 750-850 $\mathrm{nm}$. In the ternary blend PBDB-TF: HC-PCIC: PC $_{71} \mathrm{BM}$, the PL spectrum of pure PBDB$\mathrm{TF}$ is quenched almost completely, suggesting that charge separation might increase to some extent due to the faster charge transport.

Non-radiative energy loss: Xing et al. ${ }^{107}$ achieved a PCE value of $13.70 \%$ in $1.05 \mathrm{~cm}^{2}$ devices by simultaneously synergizing blade coating and adopting a ternary approach. 
A blade-coated binary device suffers a relatively high $E_{\text {loss }}$ of $0.766 \mathrm{eV} ; E_{\text {loss }}$ in a ternary solar cell is $0.752 \mathrm{eV}$ with blade coating, corresponding to a $V_{\text {oc }}$ of $0.857 \mathrm{eV}$ in the bladecoated binary device and 0.871 in the ternary device. On the one hand, the incorporation of FA elevated $V_{\text {oc }}$, from $0.857 \mathrm{eV}$ in the binary device to $0.871 \mathrm{eV}$ in the ternary device, due to the decreased non-radiative recombination loss (from $0.402 \mathrm{eV}$ to $0.367 \mathrm{eV}$ ). On the other hand, the molecular stacking was rearrangement and the crystallinity was strengthened in the ternary blend, in contrast to the corresponding binary blend, which would ameliorate charge recombination and be good for $J_{\mathrm{sc}}$ and FF to some extent.

Flexibility: There are two key parameters, device performance and mechanical properties, for flexible OSCs. As for NFA-based binary OSCs, it is common that the superior charge transport property is achieved by enhancing the crystallinity of the NFA, which is detrimental to device flexibility. Huang et al. ${ }^{108}$ found that the incorporation of proper $\mathrm{PC}_{71} \mathrm{BM}$ into the PBDTTT-OFT: IEICO-4F binary blend (PBDTTT-OFT, poly[4,8-bis(5-(2-ethylhexyl) thiophen-2-yl)benzo[1,2-b;4,5-b']dithiophene -2,6-diylalt-(4-octyl-3-fluorothieno[3,4-b]thiophene)-2-carboxylate-2-6-diyl]) may improve device performance and flexibility, simultaneously. The crystallinity of IEICO-4F decreased after doping with a proper amount of $\mathrm{PC}_{71} \mathrm{BM}$ without the formation of a rigid pure domain of $\mathrm{PC}_{71} \mathrm{BM}$, which can improve the device flexibility. In addition, the high charge transport property in the ternary device is derived predominantly from the charge transport property of $\mathrm{PC}_{71} \mathrm{BM}$. In contrast to the binary device, the exciton dissociation and charge extraction and collection are also enhanced in a ternary device. The potential of FA that can be used in semitransparent OSCs was also studied, indicating that the strategy of using ternary devices involving a FA and an NFA is very promising. ${ }^{109}$

In conclusion, the role of the FA in a ternary blend could involve a synergy of ameliorative morphology, charge dynamics, and other effects, by which optimal device efficiency can be achieved. Moreover, the introduction of a FA into a binary device based on NFA may help to simultaneously improve the performance and stability of a device, and thus improve the feasibility of commercialization. ${ }^{110-113}$

\section{Incorporation of NFAs into Fullerene-Based Binary Systems \\ Enhancing Optical Absorption}

A key bottleneck problem for binary OSCs based on fullerene derivatives is the deficient utilization of the solar spectrum because fullerene derivatives show limited and weak absorption in the short-wavelength region, leading to unsatisfactory $J_{\text {sc }}$. Due to the highly adjustable optical bandgaps, NFAs usually can significantly enhance the optical absorption of OPVs based on FA, providing an effective way to improve device performance. ${ }^{114-118}$

Tan et al. ${ }^{90}$ adopted the advisable approach to construct the absorption spectrum-complementary PBDTBDD: ${ } C_{60}$ BM: ITIC ternary device (PBDTBDD, a new copolymer based on 4,8-bis(5-(2-ethylhexyl-2-yl)benzo[1,2-b:4,5$\mathrm{b}^{\prime}$ ]-dithiophene (BDT) and 1,3-bis(thiophen-2-yl)-5,7-bis (2-ethylhexyl)benzo-[1,2-c:4,5-c'] dithiophene-4,8-dione (BDD)). The PBDTBDD: PC $_{60}$ BM: ITIC (with 40 wt\% ITIC) ternary system achieved a higher PCE (10.36\% vs. 6.63\%) and $J_{\mathrm{sc}}\left(17.76\right.$ vs. $\left.10.99 \mathrm{~mA} \mathrm{~cm}^{-2}\right)$ than the PBDTBDD: $\mathrm{PC}_{60} \mathrm{BM}$ binary OPV. The improved $J_{\text {sc }}$ was attributed to the additional absorption from ITIC. When the ratio of $\mathrm{PC}_{60} \mathrm{BM}$ : ITIC was up to 0.6:0.4, the ternary blend presented maximum photo-harvesting for the generation of photoinduced carriers. Moreover, the ternary blend displayed proper phase separation, a smooth and uniform surface that is nearly the same as the binary blend based on FA, but the domain size and roughness changed slightly, indicating that the incorporation of ITIC had a little effect on the blend morphology. By contrast, the incorporation of ITIC provides more charge transmission channels for charge transport to suppress recombination. Tan et al. ${ }^{119}$ also employed cathode engineering in ternary devices to improve device efficiency. The ternary OPV PBDTBDD: $\mathrm{PC}_{71} \mathrm{BM}$ : IEICO with zirconium acetylacetonate (ZrAcac) was used as the cathode buffer layer and achieved a PCE of $10.51 \%$. The binary blend based on PBDTBDD: $\mathrm{PC}_{71} \mathrm{BM}$ displayed an absorption spectrum in the range of $300-700 \mathrm{~nm}$, and the absorption spectrum range was extended to $900 \mathrm{~nm}$ with the addition of IEICO. In addition, they improved the cathode interfacial layer (CIL): substitution of $\mathrm{Mg}$ with $\mathrm{ZrAcac}$ reduced the series resistance and increased the photocurrent. Improvement of the contact between the active layer and CIL could be implemented by optimizing the ratio of $\mathrm{PC}_{71} \mathrm{BM}$ : IEICO, which is beneficial for decreasing contact resistance as well as increasing electron collection. As a result, $J_{\text {sc }}$ increased from $14.19 \mathrm{~mA} \mathrm{~cm}^{-2}$ in the binary OPV based on a FA to $17.98 \mathrm{~mA} \mathrm{~cm}^{-2}$ in the ternary OPV.

The ternary strategy was also implied for thick-film devices. Zhang et al. ${ }^{120}$ designed and manufactured a thickfilm ternary device $(230 \mathrm{~nm})$ with a PCE of $11.21 \%$ by incorporating the ITIC acceptor into the PDOT: $\mathrm{PC}_{71} \mathrm{BM}$ binary system (PDOT, a copolymer based on 4,8-bis(4,5-dioctylthiophen-2-yl) benzo[1,2-b:4,5-b']dithiophene-2,6-diyl-alt- $N$ (2-hexyldecyl)-5, 5'-bis(thiophen-yl)-2,2'-bithiophene-3,3'dicarboximide). The PDOT: the $\mathrm{PC}_{71} \mathrm{BM}$ binary device presented a low $J_{\mathrm{sc}}$ of $13.92 \mathrm{~mA} \mathrm{~cm}^{-2}$ because of the intrinsic narrow absorption of $300-700 \mathrm{~nm}$, but the excellent FF of $72.9 \%$ owes to the efficient charge transport property. By contrast, the PDOT: ITIC binary device was constrained by the charge transport property of ITIC, compared with the efficient 
charge transport property of $\mathrm{PC}_{71} \mathrm{BM}$. To balance the optical absorption and charge transport, Zhang et al. used $\mathrm{PC}_{71} \mathrm{BM}$ and ITIC as acceptors in a ternary solar cell, in which $\mathrm{PC}_{71} \mathrm{BM}$ and ITIC can simultaneously display their advantageous properties of outstanding electron mobility and strong optical absorption. The blend absorption was extended to $800 \mathrm{~nm}$ with the incorporation of ITIC (Figure 9). Although the EQE spectrum of the ternary blend slightly decreased in the range

(a)
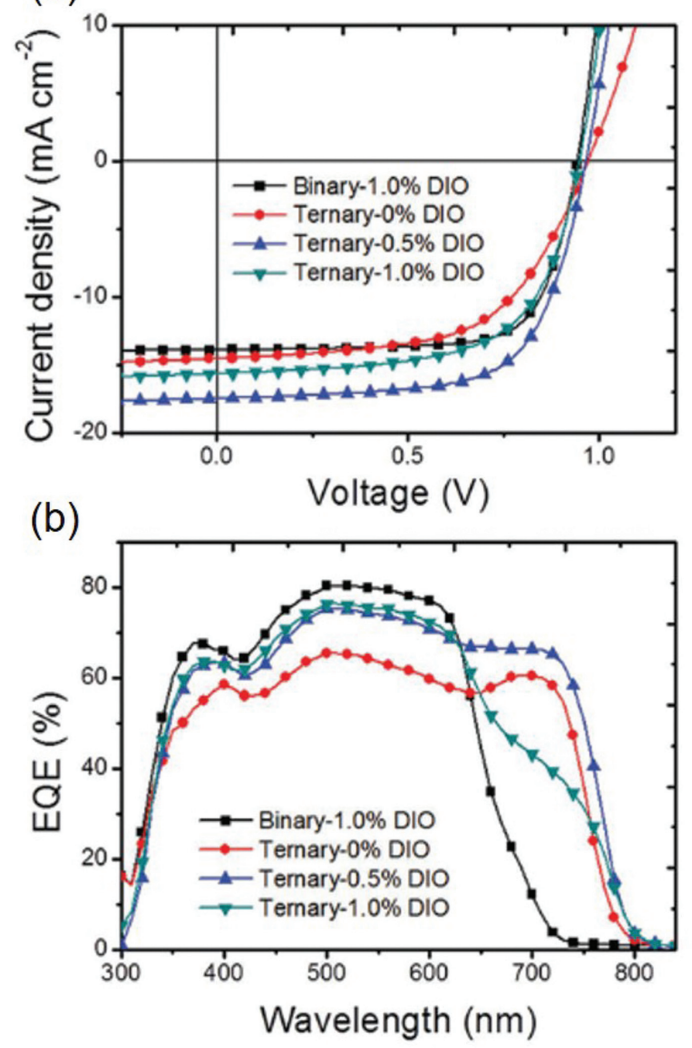

Figure 9 a) $J-V$ curves of PDOT: $\mathrm{PC}_{71} \mathrm{BM}$ binary OPVs and PDOT: $\mathrm{PC}_{71} \mathrm{BM}$ : ITIC ternary OPVs with $0 \%, 0.5 \%$, and $1.0 \% \mathrm{DIO}$; and b) their corresponding EQE spectra. Reprinted with permission from Ref. 120. Copyright 2016 Wiley-VCH.

of 300-700 nm compared with that of the ITIC-based binary solar cell due to the reduced $\mathrm{PC}_{71} \mathrm{BM}$ content, a considerable improvement in the range of 700-800 nm can be observed, which is ascribed to the direct absorption of ITIC. Consequently, optimized thick-film ternary solar cells (over $200 \mathrm{~nm}$ ) exhibited an increase in $J_{\mathrm{sc}}$ of $26 \%$ (from 13.92 to $17.49 \mathrm{~mA} \mathrm{~cm}^{-2}$ ), resulting in an increase in PCE from $9.54 \%$ to $11.21 \%$.

Bi et al. ${ }^{121}$ used an ultralow band-gap small-molecular IEICO-4F as a guest acceptor, added into a PTB7-Th: $\mathrm{PC}_{71} \mathrm{BM}$ binary system and achieved a PCE of $11.24 \%$. The improve- ment in ternary device performance was mainly ascribed to the high $J_{\mathrm{sc}}$ (from 17.39 to $23.68 \mathrm{~mA} \mathrm{~cm}^{-2}$ ), and is consistent with the enhanced EQE values in the range of 800-1000 nm, which could generate more photoinduced charge carriers by additional IEICO-4F.

Kim et al. ${ }^{92}$ fabricated the PBTTFB: PC $_{70}$ BM binary OPV (PBTTFB, a copolymer based on $N$-alkylthieno[3,4-c]pyrrole-4,6-dione (TPD) as the acceptor and BDT as the donor) with an ideal morphology, consistent with a high FF of 73\%, but it suffered insufficient absorption. They employed ITIC to improve the absorption of the PBTTFB: $\mathrm{PC}_{70} \mathrm{BM}$ binary OPV. Surprisingly, $10 \mathrm{wt} \%$ of ITIC could maximize the EQE value of the PBTTFB: ITIC: $\mathrm{PC}_{70} \mathrm{BM}(1: 0.15: 1.35)$ ternary blend and inherited the ideal morphology from the FAbased binary blend, leading to the higher $J_{\mathrm{sc}}$ of $13.73 \mathrm{~mA} \mathrm{~cm}^{-2}$ as compared with $10.79 \mathrm{~mA} \mathrm{~cm} \mathrm{~cm}^{-2}$ of the PBTTFB: $\mathrm{PC}_{70} \mathrm{BM}$ binary OPV. As the $\Delta E_{\mathrm{HOMO}}=0.05 \mathrm{eV}$ of PBTTFB and ITIC is negligible, the holes can be efficiently transferred from ITIC to PBTTFB, revealed by femtosecond TA spectroscopy, which indicates that the hole transfer is not completely decided by the difference in the HOMO energy level between the acceptor and the donor, but is significantly affected by the blend morphology. In the ternary device, the presence of $\mathrm{PC}_{70} \mathrm{BM}$ can suppress the large aggregation of ITIC and form a modified thin-film morphology, which can boost the transfer of holes and charge transport from ITIC to PBTTFB, and can inhibit bimolecular recombination. All of the above provide strong evidence that the direct incorporation of NFAs into FA-based binary solar cells can enhance absorption, then generate more photoinduced carriers, further improving $J_{\mathrm{sc}}$ and thus photovoltaic performance. This indicates that the ternary strategy is a promising candidate method for the utmost utilization of the solar spectrum.

\section{Forming a Cascade Energy Level}

In contrast to fullerene derivatives, NFAs exhibit an adjustable chemical structure with controllable energy levels, which may help form cascade energy level alignment in ternary blends to accelerate exciton dissociation and charge transfer, and further improve $J_{\mathrm{sc}}$ and FF. In this case, the NFAs are supposed to present suitable energy levels, and the HOMO and LUMO energy levels of the donor, NFA, and FA should align in a decreasing sequence. Besides, the NFAs should lay the interface between the host donor and the host acceptor and serve as an intermediate, called "bridge." The "bridge" can accelerate the charge transfer between the host donor and acceptor, and then make contributions to $J_{\mathrm{sc}}$ and $\mathrm{FF}$, and thus to device efficiency. The value of $V_{\text {oc }}$ in OSCs is mainly determined by the energy difference between the HOMO energy level of the donor and the LUMO energy level of the acceptor, and in theory the incorporation of the second 
acceptor as the "bridge" in the cascade model will have little effect on $V_{\text {oc }}$. Therefore, it offers an opportunity to simultaneously improve $J_{\mathrm{sc}}$ and $V_{\mathrm{oc}}$ via introducing NFAs with suitable energy levels as the second acceptor. In other words, the formation of a cascade energy level in ternary solar cells can provide an efficient approach to improve $J_{\mathrm{sc}}, V_{\mathrm{oc}}$, and $\mathrm{FF}$ simultaneously. The charge transfer in the cascade model can be detected by steady and transient PL measurements. If the charge transfer occurs between the two acceptors, the PL emission intensity of one acceptor would be quenched, and no obvious improvement in the PL emission intensity of other acceptor would be detected.

As mentioned before, the location of the third component in the ternary blend also plays a key role in the cascade energy alignment. Kranthiraja et al. ${ }^{122}$ studied a ternary cell consisting of an optimized P1: PC ${ }_{71} \mathrm{BM}$ : ITIC (1:1:0.1) blend (P1, poly[4,8-bis(2-(4-(2-ethylhexyloxy)3-fluorophenyl)-5thienyl)benzo[1,2-b:4,5-b']dithiophene-alt-1,3-bis(4octylthien-2-yl)-5-(2-ethylhexyl)thieno[3,4-c]pyrrole-4,6dione]), and according to the wetting coefficient value (0.086) in the ternary blend, ITIC was located at the interface between the $\mathrm{P} 1$ and the $\mathrm{PC}_{71} \mathrm{BM}$. Based on the surface energies of $\mathrm{PC}_{71} \mathrm{BM}$ : ITIC (1:1), P1: ITIC (1:1), and P1: $\mathrm{PC}_{71} \mathrm{BM}(1: 1: 0)$, represented by $\gamma_{\mathrm{PCBM}-\mathrm{ITIC}}, \gamma_{\mathrm{P} 1 \text {-ITIC, }}$ and $\gamma_{\mathrm{P} 1-}$ PCBM, respectively, the wetting coefficient of ITIC $\left(\omega_{\text {ITIC }}\right)$ in the ternary blend can be calculated via Young's equation: $\left(\omega_{\text {ITIC }}=\frac{\gamma_{\text {PCBM }- \text { ITIC }}-\gamma_{\mathrm{P} 1-\mathrm{TTC}}}{\gamma_{\mathrm{P} 1}-\mathrm{PCBM}}\right)$. The value of $\omega_{\text {ITIC }}(0.086)$ is between -1 and 1 ; thus, the location of ITIC may be at the P1/PC ${ }_{71} \mathrm{BM}$ interface, and it also can be revealed by TEM - energy dispersive X-ray analysis elemental mapping of the binary and ternary blends. Besides, $\mathrm{P} 1$, ITIC, and $\mathrm{PC}_{71} \mathrm{BM}$ display a decreasing LUMO energy level of $-3.62,-3.89$, and $-4.00 \mathrm{eV}$, respectively, as well as a deepened HOMO energy level of $-5.44,-5.49$, and $-5.68 \mathrm{eV}$, respectively. Such a cascade energy alignment would facilitate the charge separation and transfer, and the introduction of ITIC could enhance absorption, leading to an improvement in $J_{\text {sc }}$ (from 12.60 to $17.71 \mathrm{~mA} \mathrm{~cm}^{-2}$ ).

Hwang et al. ${ }^{123}$ reported that incorporation of ITIC into the PBT-OTT: $\mathrm{PC}_{71} \mathrm{BM}$ binary system [PBT-OTT, a polymer based on PBT with a 3-octylthiophene (OT) $\pi$-bridge (PBT-OT), and PBT with a 3-octylthieno[3,2-b]thiophene (OTT) $\pi$-bridge, (PBT, a copolymer based on 4,8-bis(5ethylhexylselenophen-2-yl)benzo[1,2-b:4,5-b']dithiophene (EHSeBDT) and 5-(2-butyloc-tyl)-4H-thieno[3,4-c]pyrrole4,6(5H)-dione (BOTPD)] might form a cascade energy level, and this can be revealed by the PL spectra. The emission signal of PBT-OTT was quenched without an increased PL signal of ITIC in the PBT-OTT: ITIC blend film, and the PL signal of ITIC decreased without an increased PL signal of $\mathrm{PC}_{71} \mathrm{BM}$ in the ITIC: $\mathrm{PC}_{71} \mathrm{BM}$ blend film, which suggests that efficient charge transfers occur in the ternary system. It was also found that the introduction of ITIC can facilitate charge transport and reduce charge recombination, and achieved a higher and more balanced charge mobility. Besides, there is an obvious difference in film morphology between the binary and ternary systems. The density of surface traps decreased and crystallinity increased after adding a proper amount of ITIC; the modified film morphology also made contributions to charge carrier transport, which benefits $J_{\text {sc }}$ and FF. As a result, the ternary device shows an enhancement in $J_{\mathrm{sc}}$ (from 13.3 to $14.8 \mathrm{~mA} \mathrm{~cm}{ }^{-2}$ ), $\mathrm{FF}$ (from $58.5 \%$ to $63.0 \%$ ), and PCE (from $6.74 \%$ to $8.78 \%$ ).

Other reports would also confirm that the formation of cascade energy levels in ternary blends may accelerate the charge transfer and transport by choosing NFA with a suitable energy level, in comparison with the corresponding FA-based binary blend. ${ }^{124-127}$ This approach can improve the efficiency of binary OPVs based on FA by the increased $J_{\text {sc }}$. Despite this, the improvement in PCE would not rely only on the formation of the cascade energy level. The enhanced optical absorption or modified thin-film morphology also plays a key role.

\section{Other Effects}

As discussed earlier, the incorporation of a FA into NFAbased binary OPVs can modify the active layer morphology. It is worth noting that the incorporation of NFA into FAbased binary OPVs can also increase the device efficiency via improved morphology. ${ }^{128-131}$ Moreover, the addition of an NFA may form a hydrogen bond with a FA, modify the connection between the donor and the acceptor by virtue of its tunable molecular structure, and also change the dielectric constant and reduce the Coulomb capture radius to mend charge dynamics, to further improve the device performance.

Morphology: Cheng et al. ${ }^{132}$ reported a more controlled structure in a ternary device by the sequential solution process, which is beneficial for forming the ideal vertical distribution and thus the device performance. Orthogonal solvents were used for fabricating a ternary device by spin coating for the ideal vertical distribution in the ternary active layer. At first, Cheng et al. used the PTB7-Th: $\mathrm{PC}_{71} \mathrm{BM}(1: 2)$ binary device and the PTB7-Th: PC $_{71}$ BM: IDIC (1:2:0.45) ternary device (IDCI, a planar fused-ring electron acceptor based on indacenodithiophene with four $n$-hexyl side chains) inverted device by simple spin coating, in which the donor and acceptor are simultaneously spin coated, both are limited by non-uniform vertical distribution of the materials. The mass aggregation of the donor closer to the bottom surface would impair the charge collection of the cathode. In addition, the third component would form an isolated phase in the simple ternary (S-ternary) blend OPV based on PTB7-Th: PC $_{71} \mathrm{BM}$ : IDIC (1:2:0.45), and this acted as a recombination center to impede charge transport. Thus, the S-ternary suffered poor 
mobility consistent with poor FF. To solve this issue, a more controlled structure ternary (C-ternary) was reported, in which the guest acceptor and the host D/A binary blend are sequentially spin coated by orthogonal solvents. The C-ternary blend showed an ideal distribution of the donor in the vertical direction for the more efficient charge transport and collection, as well as weaker recombination. As a result, the C-ternary blend OPV with the highest PCE exhibits improved efficiency among the binary and S-ternary blend OPVs, with increased $J_{\text {sc }}$ (from 16.3 to $18.7 \mathrm{~mA} \mathrm{~cm}^{-2}$ ), FF (from $67.2 \%$ to $69.6 \%$ ), and PCE (from 9.10\% to $10.70 \%$ ) compared with the PTB7-Th: $\mathrm{PC}_{71} \mathrm{BM}$ binary OPV. Moreover, introducing an NFA into a donor: FA may solve the issue of poor morphology in a binary blend with a FA, like forming a proper phase separation, suppressing mass aggregation, as well as adjusting the crystallinity and enlarging the D/A interface of the blend, to further benefit the charge dynamic. ${ }^{40,125,126,133,134}$

Hydrogen bond: The formation of hydrogen bonds in a ternary device can also contribute to device performance. Du et al. ${ }^{135}$ selected 3-(diethylamino)-7-imino-7H-benzo [4,5]imidazo [1,2-a]chromeno[3,2-c]pyridine-6-carbonitrile (DIBC) as the second acceptor to improve the efficiency of the PTB7-Th: PC $_{71}$ BM binary OPV. The $\mathrm{N}-\mathrm{H}$ bond in DIBC possessing an exposed $\mathrm{H}$-atom may form a hydrogen bond with the carbonyl group of $\mathrm{PC}_{71} \mathrm{BM}$ in a ternary device, which was verified by Fourier-transform infrared spectra and two-dimensional NMR. $\mathrm{PC}_{71} \mathrm{BM}$ presented a higher electrostatic potential due to the presence of an intermolecular hydrogen bond, and it enlarged the potential difference in the ternary blend for efficient exciton dissociation. In addition, the fullerene-based OPV was limited by the relatively low FF because of the mass aggregation of $\mathrm{PC}_{71} \mathrm{BM}$, but the introduction of $15 \mathrm{wt} \%$ of DIBC can reduce the phase separation into a suitable domain as well as construct the bi-continuous interpenetrating network, which may be related to the intermolecular hydrogen bond interaction. Du et al. also synthesized 3(diethylamino)-7-oxo-7H-(1)benzopyrano (3', 2':3,4) pyrido (1,2-a) benzimidazole-6-carbonitrile (DOBC), which had a similar chemical structure and absorption to DIBC, but would not form an intermolecular interaction with $\mathrm{PC}_{71} \mathrm{BM}$, leading to a decrease in PCE with poor morphology and charge dynamics compared with the PTB7-Th: $\mathrm{PC}_{71} \mathrm{BM}$ binary OPV. The PTB7-Th: $\mathrm{PC}_{71} \mathrm{BM}$ : DIBC ternary device showed an improvement of $26 \%$ in PCE (from $9.69 \%$ in the binary OPV to $12.17 \%$ in the ternary device) with increased $J_{\text {sc }}$ and $\mathrm{FF}$ (from 18.43 to $20.68 \mathrm{~mA} \mathrm{~cm}^{-2}$ and from $64.54 \%$ to $73.37 \%$, respectively).

Interfacial connection: Given the adjustable molecular configuration of NFAs, Sharapov et al. used a unique smallmolecule TPB, bearing four $\alpha$-perylenediimides (PDIs), in which the four PDIs form a cross-like molecular conformation while still being partially conjugated with the BDT-Th core to fabricate a well-performing ternary device. ${ }^{136}$ According to Young's equation, the value of $\omega_{\text {TPB }}$, the wetting coefficient of TPB in the PTB7-Th: $\mathrm{PC}_{71} \mathrm{BM}$ blend, is 0.47 , within the range between -1 and 1 , which indicates that TPB may largely be at the interface of PTB7-Th: PC $_{71}$ BM. Thus, TPB can act as an electronic relay bridge between the polymer and $\mathrm{PC}_{71} \mathrm{BM}$ domains for better connectivity between the two phases, leading to an increased charge generation. An efficient electron transfer occurs between TPB and $\mathrm{PC}_{71} \mathrm{BM}$, which may be ascribed to TPB with a crosslike molecular structure, which can offer more contact sites between TPB and $\mathrm{PC}_{71} \mathrm{BM}$, and to the LUMO energy levels that are nearly equal for $\mathrm{PC}_{71} \mathrm{BM}$. Hence, the strongly enhanced electron mobility could improve the value of $J_{\mathrm{sc}}$ in the ternary system compared with the binary system. In light of GIWAXS images, the fullerene-based binary blend exhibits amorphous characteristics, whereas incorporation of TPB would strengthen the crystallinity of PTB7-Th: $\mathrm{PC}_{71} \mathrm{BM}$ blend for better charge transport and extraction. Therefore, $J_{\mathrm{sc}}$ increased from $17.2 \mathrm{~mA} \mathrm{~cm}^{-2}$ in the PTB7-Th: $\mathrm{PC}_{71} \mathrm{BM}$ binary device to $19.4 \mathrm{~mA} \mathrm{~cm} \mathrm{~cm}^{-2}$ in the PTB7-Th: $\mathrm{PC}_{71} \mathrm{BM}$ : TPB (1:1.5:0.1) ternary device. To summarize, Sharapov et al. manufactured a ternary OPV device with a higher PCE of $10.6 \%$ compared with $9.8 \%$ in a fullerenebased binary OPV.

Dielectric constant and Coulomb capture radius: Duan et al. demonstrated that the introduction of an NFA, poly[[4,8-bis[(2-ethylhexyl)oxy]benzo[1,2-b:4,5-b']dithiophene-2,6-diy-1][3-fluoro-2-[(2-ethylhexyl)carbonyl] thieno[3,4-b]thiophene-diyl (IDFBR) into a binary blend of PTB7: $\quad$ PC $_{71} \mathrm{BM}$ (PTB7, poly[[4,8-bis[(2-ethylhexyl)oxy] benzo[1,2-b:4,5-b'] dithiophene -2,6-diyl][3-fluoro-2-[(2ethylhexyl)carbonyl]thieno[3, 4-b]thiophenediyl]]) can efficiently improve device performance by adjusting the dielectric constant and reducing the Coulomb capture radius $^{137}$; the former is related to the exciton binding energy and charge carrier recombination, and the latter is associated with charge generation. Duan et al. fabricated a series of ternary OPVs (PTB7: $\mathrm{PC}_{71} \mathrm{BM}$ : IDFBR) with varying amounts of $\operatorname{IDFBR}(0,5,10,15$, and $100 \mathrm{wt} \%)$, and the device PTB7: PC $_{71}$ BM: IDFBR (with 5 wt\% IDFBR) showed the highest PCE. The absorption curve of the ternary blend with $5 \mathrm{wt} \%$ IDFBR improved in the range of $450-800 \mathrm{~nm}$ compared with the binary blend, but was lower than that of the ternary blend with 10 and 15 wt\% IDFBR. Nevertheless, the ternary blend with $5 \mathrm{wt} \%$ IDFBR displayed maximum EQE values, which may be attributed to increased dielectric constant and decreased Coulomb capture radius, leading to enhanced charge separation and transport, as well as reduced charge recombination, consistent with the increase in $J_{\mathrm{sc}}$ (from 16.70 to $17.38 \mathrm{~mA} \mathrm{~cm}^{-2}$ ) and $\mathrm{FF}$ (from $50.63 \%$ to $55.56 \%$ ). Furthermore, the LUMO/HOME energy level of IDFBR was in between those of PTB7 and $\mathrm{PC}_{71} \mathrm{BM}$, which was responsible for the improved charge transport, 
and thus the value of $V_{\text {oc }}$ (from 0.716 to $0.731 \mathrm{~V}$ ). In summary, the strategy of using a ternary compound can elevate $V_{\mathrm{oc}}, J_{\mathrm{sc}}$, and FF with an improvement of $12 \%$ in PCE compared with the corresponding binary device.

Charge dynamics: The addition of NFA into a FA-based binary device may also amend its charge dynamics. A report found via theoretical calculations showed that combining IT-M with the PTB7-Th: $\mathrm{PC}_{71} \mathrm{BM}$ binary blend may facilitate charge transport. ${ }^{138}$ In other words, the incorporation of NFAs into binary devices based on FA is a promising strategic method to realize high-performance OPVs.

\section{Balanced Charge Separation and Transport Enabled by Ideal Hierarchical Morphology}

The film morphology of an active layer has a significant effect on the performance of OPVs. Most reports on ternary OSCs fail to explain how the changes in morphology affect the charge dynamics to further improve device performance, which is similar to conventional analysis of binary OSCs and is unable to make full use of the merits of a strategy of using a ternary compound in OPV devices. For instance, although there is morphological amelioration in crystallinity in a binary system, transverse and vertical phase separation can be achieved via the "ternary strategy"; it can also be realized by device optimization in binary OSCs. Hence, in this section, ternary systems for morphology control according to the hierarchical structure are discussed in detail.

In general, charge generation needs small phase length, while efficient charge transport needs a continuous fibrous network framework. Both of them are hardly achieved in the active layer with uniformity domain, simultaneously. Nevertheless, the hierarchical morphology with multiple phase length can balance the charge generation and transport for an optimal device performance. The formation of a hierarchical morphology consisting of multiple phases with a complex multilength scale in the ternary system may be an instructive strategy to figure out the relationship between morphology and performance of OSCs, in which the small phase separation is responsible for exciton diffusion and charge separation and the large phase separation can boost charge carrier transport and collection and thus device performance.

Our group first utilized FA ( $\left.\mathrm{PC}_{71} \mathrm{BM}\right)$ and NFA (NITI) and a small molecular donor (BTR, BDT terthiophene rhodamine; NITI, 2,2'-((2Z,2'Z)-((6,6'-(5,5,10,10-tetrakis(2-ethylhexyl)5,10-dihydroindeno[2,1-a]indene-2,7-diyl)bis(2-octylthieno[3,4-b]thiophene-6,4-diyl))bis(methanylylidene))bis (5,6-difluoro-3-oxo-2,3-dihydro-1H-indene-2,1-diylidene)) dim-alononitrile) in a ternary system, leading to highperformance OPV with a hierarchical morphology. ${ }^{129}$ The high-efficiency ternary OPV can greatly balance the charge carrier generation and transport and fully utilize the merits of the ternary strategy. The hierarchical morphology consists of the $\mathrm{PC}_{71} \mathrm{BM}$ framework fitted by a refined phase separation of BTR: NITI, and more specifically, the NITI lies between PC $_{71} B M$ and BTR with a preferred face-on orientation towards the $\mathrm{PC}_{71} \mathrm{BM}$ (Figure 10). On the one hand, the fine BTR: NITI phase
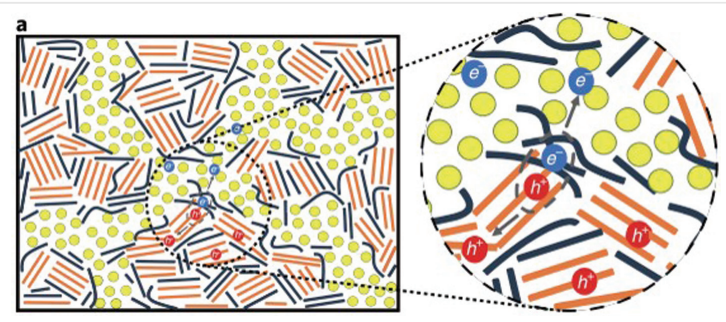

Figure 10 a) The BTR (orange rods): PC $_{71} \mathrm{BM}$ (green circles): NITI (navy rods) ternary blend film with ideal hierarchical morphology. Reprinted with permission from Ref. 129. Copyright 2018 Springer Nature.

separation can offer sufficient D/A interface for efficient exciton dissociation. On the other hand, the presence of $\mathrm{PC}_{71} \mathrm{BM}$ can boost charge transport and extraction, which is revealed by the results of the dependence of photocurrent density on the effective voltage among the ternary and two binary OPVs. Besides, the NITI can receive electron from the $\mathrm{BTR}$ and then fast transfer it to the $\mathrm{PC}_{71} \mathrm{BM}$ for the demand of a passivating energetic disorder. In consequence, the trapassisted recombination reduced in the ternary blend with the incorporation of NITI. From the investigation of electroluminescence spectroscopy, our group found that the existence of NITI would interdict the contact between $\mathrm{PC}_{71} \mathrm{BM}$ and BTR to reduce non-radiative energy loss. Thus, such a ternary OSC with ideal hierarchical morphology can maximize the values of $V_{\mathrm{oc}}, J_{\mathrm{Sc}}$ and FF, and achieve a high PCE of $13.63 \%$ in the BTR: NITI: $\mathrm{PC}_{71} \mathrm{BM}$ ternate device. Our group also expanded this research to other small-donor (DR3TSBDT/DR3TBDTT)/FA $\left(\mathrm{PC}_{71} \mathrm{BM}\right) / \mathrm{NFA}$ (ITIC/NITI-EH) ternary systems and realized the possible formation progress of a hierarchical morphology. The precondition of a hierarchical morphology is the formation of refined donor: NFA phase separation and large-scale mixing between $\mathrm{PC}_{71} \mathrm{BM}$ and other components. The large $\mathrm{PC}_{71} \mathrm{BM}$ framework formed via phase separation, firstly. The crystallinity of the donor domain enhanced during the solvent vapour annealing. Then the NFA squeezed out from the donor domain and congregated in the interface of the $\mathrm{PC}_{71} \mathrm{BM}$ framework and formed small-size phase separation. The high-performance device can be attributed to the formation of a hierarchical morphology, and it was also demonstrated by Wei's group. ${ }^{139}$ The combination of fullerene and NFAs has shown great potential in achieving record OPV performance. ${ }^{17,19,42,96,118,140,141}$ 


\section{Conclusions and Outlook}

The above-mentioned discussion was meant to clarify that the ternary device synergizing a FA and an NFA can overcome the limitations of conventional OPVs based on a single D-A pair, as well as benefit from its advantages. The incorporation of FA into the binary blend based on NFA can promote the device efficiency by rearranging and suppressing the molecular stacking and mass aggregation of the host $\mathrm{D} / \mathrm{A}$, adjusting the crystallinity and vertical distribution of the active layer in the ternary device, forming bi-continuous interpenetrating network or forming energy transfer. As for binary OPVs based on FA, the addition of a NFA mainly acts as a light absorber or forms cascade energy level alignment. Surprisingly, it can also be a morphology modifier to improve device efficiency. Moreover, the ternary strategy may improve the long-term stability of the device by maintaining the morphology of the active layer. Through numerous research studies, it is firmly believed that the ternary strategy based on NFA and FA is a promising way to obtain high-performance OSCs, because it can efficiently combine the advantages of both NFA and FAs along with overcoming the respective shortcomings of each type. Recently, Liu et al. synergized $\mathrm{Y} 6$ and $\mathrm{PC}_{71} \mathrm{BM}$ as acceptors combined with the PM6 and PM7 (poly[(2,6-(4,8-bis(5-(2ethylhexyl)-4-chlorothiophen-2-yl)benzo[1,2-b:4,5-b'] dithiophene))-co-(1,3-di(5-thiophene-2-yl)-5,7-bis(2-ethylhexyl )-benzo[1,2-c:4,5-c'] dithiophene-4,8-dione))]) donor pair, and achieved a device with a high PCE of over $18 \%$ with ameliorative charge kinetics and reduced non-radiation loss via a double cascading charge transport, which can demonstrate that the multicomponent strategy can effectively improve device performance. ${ }^{141}$ The ternary solar cell has reached a high performance of $18.3 \%$ via the synergy effect of n-doping and the ternary strategy. ${ }^{19}$ In addition, this ternary strategy can also efficiently improve device stability. ${ }^{13}$ And much research has been conducted to achieve the higher performance OSCs via the use of a ternary component (ternary strategy) ${ }^{141-147}$ and even a quaternary component (quaternary strategy). ${ }^{148}$ However, the specific working mechanism of ternary OPVs is debatable owing to the complexity of ternary OPVs that use three different components in a single device. Thus, the working mechanism of ternary OPVs need to be further explored.

Despite the many recent successful examples and inspiring advances in ternary OPVs reported above, some fundamental issues still need to be addressed. (1) New materials with high absorption coefficients and excellent charge transport properties are supposed to be designed and synthesized to maximize the use of solar energy. (2) The selection of the third component in ternary OSCs should be deliberate to maximize all the aforementioned possible advantages of the ternary strategy. (3) The technologies of device fabrication that favor the stability and performance of devices need to be improved, such as the design of the buffer layer, which also is a basic premise of large-scale production for commercial application. (4) Methods that reveal the relationship between structure and performance of the ternary device should be explored to get a better understanding of the working mechanism of ternate OPVs. In summary, the ternary strategy provides a promising way to improve device efficiency and stability simultaneously, and there is still much room for it to be improved.

\section{Funding Information}

The authors thank the National Key R\&D Program of China (2019YFA0705900 and 2017YFA0204701) and the National Natural Science Foundation of China (21572234, 2166 1132006, and 91833304) for their financial support.

\section{References}

(1) Mazzio, K. A.; Luscombe, C. K. Chem. Soc. Rev. 2015, 44, 78.

(2) Wadsworth, A.; Moser, M.; Marks, A.; Little, M. S.; Gasparini, N.; Brabec, C. J.; Baran, D.; McCulloch, I. Chem. Soc. Rev. 2019, 48, 1596.

(3) Wu, J.-S.; Cheng, S.-W.; Cheng, Y.-J.; Hsu, C.-S. Chem. Soc. Rev. 2015, 44, 1113.

(4) Li, Y. Acc. Chem. Res. 2012, 45, 723.

(5) Hou, J.; Inganäs, O.; Friend, R. H.; Gao, F. Nat. Mater. 2018, 17, 119.

(6) Qian, D.; Zheng, Z.; Yao, H.; Tress, W.; Hopper, T. R.; Chen, S.; Li, S.; Liu, J.; Chen, S.; Zhang, J.; Liu, X.-K.; Gao, B.; Ouyang, L.; Jin, Y.; Pozina, G.; Buyanova, I. A.; Chen, W. M.; Inganäs, O.; Coropceanu, V.; Bredas, J.-L.; Yan, H.; Hou, J.; Zhang, F.; Bakulin, A. A.; Gao, F. Nat. Mater. 2018, 17, 703.

(7) Lou, S. J.; Szarko, J. M.; Xu, T.; Yu, L.; Marks, T. J.; Chen, L. X. J. Am. Chem. Soc. 2011, 133, 20661.

(8) Deng, D.; Zhang, Y.; Zhang, J.; Wang, Z.; Zhu, L.; Fang, J.; Xia, B.; Wang, Z.; Lu, K.; Ma, W.; Wei, Z. Nat. Commun. 2016, 7, 13740.

(9) Vohra, V.; Kawashima, K.; Kakara, T.; Koganezawa, T.; Osaka, I.; Takimiya, K.; Murata, H. Nat. Photonics 2015, 9, 403.

(10) Zhang, Y.; Deng, D.; Wang, Z.; Wang, Y.; Zhang, J.; Fang, J.; Yang, Y.; Lu, G.; Ma, W.; Wei, Z. Adv. Energy Mater. 2017, 7, 1701548

(11) Po, R.; Carbonera, C.; Bernardi, A.; Camaioni, N. Energy Environ. Sci. 2011, 4, 285.

(12) Zhao, F.; Wang, Z.; Zhang, J.; Zhu, X.; Zhang, Y.; Fang, J.; Deng, D.; Wei, Z.; Li, Y.; Jiang, L.; Wang, C. Adv. Energy Mater. 2016, 6, 1502120.

(13) Menke, S. M.; Holmes, R. J. Energy Environ. Sci. 2014, 7, 499.

(14) Lu, L.; Zheng, T.; Wu, Q.; Schneider, A. M.; Zhao, D.; Yu, L. Chem. Rev. 2015, 115, 12666.

(15) Lyons, B. P.; Clarke, N.; Groves, C. Energy Environ. Sci. 2012, 5, 7657.

(16) Venkatesan, S.; Chen, J.; Ngo, E. C.; Dubey, A.; Khatiwada, D.; Zhang, C.; Qiao, Q. Nano Energy 2015, 12, 457.

(17) Lin, Y.; Firdaus, Y.; Isikgor, F. H.; Nugraha, M. I.; Yengel, E.; Harrison, G. T.; Hallani, R.; El-Labban, A.; Faber, H.; Ma, C.; Zheng, X.; Subbiah, A.; Howells, C. T.; Bakr, O. M.; McCulloch, I.; Wolf, S. D.; Tsetseris, L.; Anthopoulos, T. D. ACS Energy Lett. 2020, 5, 2935. 
(18) Liu, M.; Fan, P.; Hu, Q.; Russell, T. P.; Liu, Y. Angew. Chem. Int. Ed. Engl. 2020, 59, 18131.

(19) Lin, Y.; Nugraha, M. I.; Firdaus, Y.; Scaccabarozzi, A. D.; Aniés, F.; Emwas, A.-H.; Yengel, E.; Zheng, X.; Liu, J.; Wahyudi, W.; Yarali, E.; Faber, H.; Bakr, O. M.; Tsetseris, L.; Heeney, M.; Anthopoulos, T. D. ACS Energy Lett. 2020, 5, 3663.

(20) Li, X.; Pan, M.-A.; Lau, T.-K.; Liu, W.; Li, K.; Yao, N.; Shen, F.; Huo, S.; Zhang, F.; Wu, Y.; Li, X.; Lu, X.; Yan, H.; Zhan, C. Chem. Mater. 2020, 32, 5182.

(21) Chen, C.; Liu, W.; Guan, X.; Zhang, J.; Yang, Q.; Qin, D. Phys. Status Solidi A 2020, 217, 2000320.

(22) Wu, Z.; Lee, S.; Jeong, S. Y.; Jee, M. H.; Lee, H. G.; Lim, C.; Wang, C.; Kim, B. J.; Woo, H. Y. Mater. Today Energy 2021, 20, 100651.

(23) Zhang, J.; Liu, W.; Xu, S.; Zhu, X. Org. Mater. 2019, 1, 30.

(24) Liu, T.; Troisi, A. Adv. Mater. 2013, 25, 1038.

(25) Chen, W.; Zhang, Q. J. Mater. Chem. C 2017, 5, 1275.

(26) Sun, H.; Song, X.; Xie, J.; Sun, P.; Gu, P.; Liu, C.; Chen, F.; Zhang, Q.; Chen, Z. K.; Huang, W. ACS Appl. Mater. Interfaces 2017, 9, 29924.

(27) Chen, W.; Yang, X.; Long, G.; Wan, X.; Chen, Y.; Zhang, Q. J. Mater. Chem. C 2015, 3, 4698.

(28) Havinga, E. E.; ten Hoeve, W.; Wynberg, H. Polym. Bull. 1992, 29, 119.

(29) Havinga, E. E.; ten Hoeve, W.; Wynberg, H. Synth. Met. 1993, 55, 299.

(30) Lin, Y.; Li, T.; Zhao, F.; Han, L.; Wang, Z.; Wu, Y.; He, Q.; Wang, J.; Huo, L.; Sun, Y.; Wang, C.; Ma, W.; Zhan, X. Adv. Energy Mater. 2016, 6, 1600854.

(31) Wang, W.; Yan, C.; Lau, T.-K.; Wang, J.; Liu, K.; Fan, Y.; Lu, X.; Zhan, X. Adv. Mater. 2017, 29, 1701308.

(32) Li, Y.; Zhong, L.; Gautam, B.; Bin, H.-J.; Lin, J.-D.; Wu, F.-P.; Zhang, Z.; Jiang, Z.-Q.; Zhang, Z.-G.; Gundogdu, K.; Li, Y.; Liao, L.S. Energy Environ. Sci. 2017, 10, 1610.

(33) Zhou, H.; Yang, L.; Stuart, A. C.; Price, S. C.; Liu, S.; You, W. Angew. Chem. Int. Ed. 2011, 50, 2995.

(34) Pan, L.; Liu, T.; Wang, J.; Ye, L.; Luo, Z.; Ma, R.; Pang, S.; Chen, Y.; Ade, H.; Yan, H.; Duan, C.; Huang, F.; Cao, Y. Chem. Mater. 2020, 32, 7309.

(35) Fan, B.; Zeng, Z.; Zhong, W.; Ying, L.; Zhang, D.; Li, M.; Peng, F.; Li, N.; Huang, F.; Cao, Y. ACS Energy Lett. 2019, 4, 2466.

(36) Zhao, C.; Wang, J.; Zhao, X.; Du, Z.; Yang, R.; Tang, J. Nanoscale 2021, 13, 2181.

(37) Cheng, P.; Zhang, M.; Lau, T. K.; Wu, Y.; Jia, B.; Wang, J.; Yan, C.; Qin, M.; Lu, X.; Zhan, X. Adv. Mater. 2017, 29, 1605216.

(38) Hu, D.; Yang, Q.; Chen, H.; Wobben, F.; Le Corre, V. M.; Singh, R.; Liu, T.; Ma, R.; Tang, H.; Koster, L. J. A.; Duan, T.; Yan, H.; Kan, Z.; Xiao, Z.; Lu, S. Energy Environ. Sci. 2020, 13, 2134.

(39) Liu, T.; Guo, Y.; Yi, Y.; Huo, L.; Xue, X.; Sun, X.; Fu, H.; Xiong, W.; Meng, D.; Wang, Z.; Liu, F.; Russell, T. P.; Sun, Y. Adv. Mater. 2016, 28, 10008.

(40) Zhang, H.; Wang, X.; Yang, L.; Zhang, S.; Zhang, Y.; He, C.; Ma, W.; Hou, J. Adv. Mater. 2017, 29, 1703777.

(41) Gao, H.-H.; Sun, Y.; Cai, Y.; Wan, X.; Meng, L.; Ke, X.; Li, S.; Zhang, Y.; Xia, R.; Zheng, N.; Xie, Z.; Li, C.; Zhang, M.; Yip, H.-L.; Cao, Y.; Chen, Y. Adv. Energy Mater. 2019, 9, 1901024.

(42) Zhu, C.; Yuan, J.; Cai, F.; Meng, L.; Zhang, H.; Chen, H.; Li, J.; Qiu, B.; Peng, H.; Chen, S.; Hu, Y.; Yang, C.; Gao, F.; Zou, Y.; Li, Y. Energy Environ. Sci. 2020, 13, 2459.

(43) Zhang, M.; Zhang, Z.; Wang, J.; An, Q.; Peng, H.; Tang, W.; Zhang, F. Sol. RRL 2019, 3, 1900269.
(44) Lu, H.; Zhang, J.; Chen, J.; Liu, Q.; Gong, X.; Feng, S.; Xu, X.; Ma, W.; Bo, Z. Adv. Mater. 2016, 28, 9559.

(45) Lu, H.; Li, M.; Bi, Z.; Gong, X.; Li, G.; Feng, S.; Xu, X.; Ma, W.; Bo, Z. Org. Electron. 2019, 65, 419.

(46) Zhang, C. e.; Feng, S.; Liu, Y.; Ming, S.; Lu, H.; Ma, D.; Xu, X.; Wu, Y.; Bo, Z. J. Mater. Chem. A 2018, 6, 6854.

(47) Schmidt, R.; Oh, J. H.; Sun, Y. S.; Deppisch, M.; Krause, A. M.; Radacki, K.; Braunschweig, H.; Könemann, M.; Erk, P.; Bao, Z.; Würthner, F. J. Am. Chem. Soc. 2009, 131, 6215.

(48) Zhang, C. e.; Jiang, P.; Zhou, X.; Liu, H.; Guo, Q.; Xu, X.; Liu, Y.; Tang, Z.; Ma, W.; Bo, Z. J. Mater. Chem. A 2020, 8, 2123.

(49) Xu, C.; Wang, J.; An, Q.; Ma, X.; Hu, Z.; Gao, J.; Zhang, J.; Zhang, F. Nano Energy 2019, 66, 104119.

(50) Qiu, B.; Chen, S.; Sun, C.; Yuan, J.; Zhang, X.; Zhu, C.; Qin, S.; Meng, L.; Zhang, Y.; Yang, C.; Zou, Y.; Li, Y. Sol. RRL 2020, 4, 1900540.

(51) Kang, Z.; Ma, Y.; Zheng, Q. Dyes Pigm. 2019, 170, 107555.

(52) Wang, B.; Fu, Y.; Yan, C.; Zhang, R.; Yang, Q.; Han, Y.; Xie, Z. Front. Chem. 2018, 6, 198.

(53) Chen, Y.; Qin, Y.; Wu, Y.; Li, C.; Yao, H.; Liang, N.; Wang, X.; Li, W.; Ma, W.; Hou, J. Adv. Energy Mater. 2017, 7, 1700328.

(54) Xie, Y.; Yang, F.; Li, Y.; Uddin, M. A.; Bi, P.; Fan, B.; Cai, Y.; Hao, X.; Woo, H. Y.; Li, W.; Liu, F.; Sun, Y. Adv. Mater. 2018, 30, e1803045.

(55) Xue, C.; Zhang, T.; Ma, K.; Wan, P.; Hong, L.; Xu, B.; An, C. Macromol. Rapid Commun. 2019, 40, 1900246.

(56) Hadmojo, W. T.; Wibowo, F. T. A.; Lee, W.; Jang, H. K.; Kim, Y.; Sinaga, S.; Park, M.; Ju, S. Y.; Ryu, D. Y.; Jung, I. H.; Jang, S. Y. Adv. Funct. Mater. 2019, 29, 1808731.

(57) Fu, H.; Li, C.; Bi, P.; Hao, X.; Liu, F.; Li, Y.; Wang, Z.; Sun, Y. Adv. Funct. Mater. 2019, 29, 1807006.

(58) Yan, T.; Song, W.; Huang, J.; Peng, R.; Huang, L.; Ge, Z. Adv. Mater. 2019, 31, 1902210.

(59) Dai, S.; Li, T.; Wang, W.; Xiao, Y.; Lau, T. K.; Li, Z.; Liu, K.; Lu, X.; Zhan, X. Adv. Mater. 2018, 30, 1706571.

(60) Yi, Y.-Q.-Q.; Feng, H.; Zheng, N.; Ke, X.; Kan, B.; Chang, M.; Xie, Z.; Wan, X.; Li, C.; Chen, Y. Chem. Mater. 2019, 31, 904.

(61) Huang, D.; Bian, F.; Zhu, D.; Bao, X.; Hong, C.; Zhou, P.; Huang, Y.; Yang, C. J. Phys. Chem. C 2019, 123, 14976.

(62) Liang, Z.; Tong, J.; Li, H.; Wang, Y.; Wang, N.; Li, J.; Yang, C.; Xia, Y. J. Mater. Chem. A 2019, 7, 15841.

(63) Zhang, K.-N.; Jiang, Z.-N.; Wang, T.; Niu, M.-S.; Feng, L.; Qin, C.C.; So, S.-K.; Hao, X.-T. Sol. RRL 2020, 4, 2000165.

(64) Chen, Y.; You, G.; Zou, D.; Zhuang, Q.; Zhen, H.; Ling, Q. Sol. Energy 2019, 183, 350.

(65) Huang, G.; Zhang, J.; Uranbileg, N.; Chen, W.; Jiang, H.; Tan, H.; Zhu, W.; Yang, R. Adv. Energy Mater. 2018, 8, 1702489.

(66) Gao, H. H.; Sun, Y.; Wan, X.; Ke, X.; Feng, H.; Kan, B.; Wang, Y.; Zhang, Y.; Li, C.; Chen, Y. Adv. Sci. 2018, 5, 1800307.

(67) Liu, Z.-X.; Lau, T.-K.; Zhou, G.; Li, S.; Ren, J.; Das, S. K.; Xia, R.; Wu, G.; Zhu, H.; Lu, X.; Yip, H.-L.; Chen, H.; Li, C.-Z. Nano Energy 2019, 63, 103807.

(68) Huo, Y.; Gong, X.-T.; Lau, T.-K.; Xiao, T.; Yan, C.; Lu, X.; Lu, G.; Zhan, X.; Zhang, H.-L. Chem. Mater. 2018, 30, 8661.

(69) Zhao, W.; Li, S.; Zhang, S.; Liu, X.; Hou, J. Adv. Mater. 2017, 29, 1604059.

(70) Liu, S.; Chen, D.; Hu, X.; Xing, Z.; Wan, J.; Zhang, L.; Tan, L.; Zhou, W.; Chen, Y. Adv. Funct. Mater. 2020, 30, 2003223.

(71) He, S.; Shen, Z.; Yu, J.; Guan, H.; Lu, G.; Xiao, T.; Yang, S.; Zou, Y.; $\mathrm{Bu}, \mathrm{L}$. Adv. Mater. Interfaces 2020, 7, 2000577.

(72) Bi, P.; Xiao, T.; Yang, X.; Niu, M.; Wen, Z.; Zhang, K.; Qin, W.; So, S. K.; Lu, G.; Hao, X.; Liu, H. Nano Energy 2018, 46, 81. 
(73) Lee, J.; Lee, J.-H.; Yao, H.; Cha, H.; Hong, S.; Lee, S.; Kim, J.; Durrant, J. R.; Hou, J.; Lee, K. J. Mater. Chem. A 2020, 8, 6682.

(74) Park, H. S.; Han, Y. W.; Lee, H. S.; Jeon, S. J.; Moon, D. K. ACS Appl. Mater. 2020, 3, 3745.

(75) Pan, M.-A.; Lau, T.-K.; Tang, Y.; Wu, Y.-C.; Liu, T.; Li, K.; Chen, M.C.; Lu, X.; Ma, W.; Zhan, C. J. Mater. Chem. A 2019, 7, 20713.

(76) Geng, R.; Song, X.; Feng, H.; Yu, J.; Zhang, M.; Gasparini, N.; Zhang, Z.; Liu, F.; Baran, D.; Tang, W. ACS Energy Lett. 2019, 4, 763.

(77) Huang, J.; Peng, R.; Xie, L.; Song, W.; Hong, L.; Chen, S.; Wei, Q.; Ge, Z. J. Mater. Chem. A 2019, 7, 2646.

(78) Liang, R.-Z.; Zhang, Y.; Savikhin, V.; Babics, M.; Kan, Z.; Wohlfahrt, M.; Wehbe, N.; Liu, S.; Duan, T.; Toney, M. F.; Laquai, F.; Beaujuge, P. M. Adv. Energy Mater. 2019, 9, 1802836.

(79) Xiao, Z.; Jia, X.; Ding, L. Sci. Bull. 2017, 62, 1562.

(80) Jiang, K.; Wei, Q.; Lai, J. Y. L.; Peng, Z.; Kim, H. K.; Yuan, J.; Ye, L.; Ade, H.; Zou, Y.; Yan, H. Joule 2019, 3, 3020.

(81) Liu, Q.; Toudert, J.; Ciammaruchi, L.; Martínez-Denegri, G.; Martorell, J. J. Mater. Chem. A 2017, 5, 25476.

(82) Wang, H.; Zhang, Z.; Yu, J.; Lin, P. C.; Chueh, C. C.; Liu, X.; Guang, S.; Qu, S.; Tang, W. ACS Appl. Mater. Interfaces 2020, 12, 21633.

(83) Privado, M.; Malhotra, P.; de la Cruz, P.; Singhal, R.; Cerdá, J.; Aragó, J.; Ortí, E.; Sharma, G. D.; Langa, F. Sol. RRL 2020, 4 , 1900471.

(84) Kang, J.; Kim, J.; Eom, S. H.; Yoon, S. C.; Jung, I. H. ACS Appl. Energy Mater. 2020, 3, 5313.

(85) Yi, Y.-Q.-Q.; Feng, H.; Ke, X.; Yan, J.; Chang, M.; Wan, X.; Li, C.; Chen, Y. J. Mater. Chem. C 2019, 7, 4013.

(86) Chang, S.-L.; Cao, F.-Y.; Huang, K.-H.; Lee, W.-L.; Lee, M.-H.; Hsu, C.-S.; Cheng, Y.-J. J. Mater. Chem. A 2020, 8, 12141.

(87) Shi, H.; Xia, R.; Zhang, G.; Yip, H.-L.; Cao, Y. Adv. Energy Mater. 2019, 9, 1803438.

(88) Wang, Z.; Ji, J.; Lin, W.; Yao, Y.; Zheng, K.; Liang, Z. Adv. Funct. Mater. 2020, 2001564.

(89) Li, Q.; Sun, Y.; Xue, X.; Yue, S.; Liu, K.; Azam, M.; Yang, C.; Wang, Z.; Tan, F.; Chen, Y. ACS Appl. Mater. Interfaces 2019, 11, 3299.

(90) Liu, H.; Li, J.; Xia, L.; Bai, Y.; Hu, S.; Liu, J.; Liu, L.; Hayat, T.; Alsaedi, A.; Tan, Z. ACS Appl. Mater. Interfaces 2018, 10, 29831.

(91) Karuthedath, S.; Firdaus, Y.; Liang, R. Z.; Gorenflot, J.; Beaujuge, P. M.; Anthopoulos, T. D.; Laquai, F. Adv. Energy Mater. 2019, 9, 1901443.

(92) Kim, T.; Heo, J.; Lee, J. Y.; Yoon, Y. J.; Lee, T. H.; Shin, Y. S.; Kim, I. S.; Kim, H.; Jeong, M. S.; Hwang, I. W.; Walker, B.; Jo, P. S.; Lim, B.; Kim, J. Y. ACS Appl. Mater. Interfaces 2019, 11, 7208.

(93) Yu, S.; Yang, Q.; Yu, W.; Zhang, J.; Liu, J.; Jin, S.; Guo, X.; Li, C. Chem. Mater. 2019, 31, 7650.

(94) Cao, F.-Y.; Huang, P.-K.; Su, Y.-C.; Huang, W.-C.; Chang, S.-L.; Hung, K.-E.; Cheng, Y.-J. J. Mater. Chem. A 2019, 7, 17947.

(95) Xue, Y.-J.; Cao, F.-Y.; Huang, P.-K.; Su, Y.-C.; Cheng, Y.-J.J. Mater. Chem. A 2020, 8, 5315.

(96) Luo, Z.; Sun, R.; Zhong, C.; Liu, T.; Zhang, G.; Zou, Y.; Jiao, X.; Min, J.; Yang, C. Sci. China Chem. 2020, 63, 361.

(97) Yu, R.; Yao, H.; Cui, Y.; Hong, L.; He, C.; Hou, J. Adv. Mater. 2019, 31, 1902302.

(98) Ma, L.; Xu, Y.; Zu, Y.; Liao, Q.; Xu, B.; An, C.; Zhang, S.; Hou, J. Sci. China Chem. 2019, 63, 21.

(99) Sun, Y.; Yang, C.; Li, Q.; Liu, K.; Xue, X.; Zhang, Y.; Azam, M.; Ren, K.; Chen, Y.; Wang, Z.; Qu, S.; Wang, Z. J. Power Sources 2020 , 449, 227583.

(100) Chang, S. L.; Cao, F. Y.; Huang, W. C.; Huang, P. K.; Hsu, C. S.; Cheng, Y. J. ACS Appl. Mater. Interfaces 2017, 9, 24797.

(101) Liu, Z.; Wang, N. J. Power Sources 2020, 448, 227442.
(102) Privado, M.; Seco, C. R.; Singhal, R.; Cruz, P. 1.; Langa, F.; Sharma, G. D.; Palomares, E. ACS Energy Lett. 2018, 3, 2418.

(103) Liu, Q.; Jiang, Y.; Jin, K.; Qin, J.; Xu, J.; Li, W.; Xiong, J.; Liu, J.; Xiao, Z.; Sun, K.; Yang, S.; Zhang, X.; Ding, L. Sci. Bull. 2020, 65, 272.

(104) Zhang, C.-H.; Wang, W.; Huang, W.; Wang, J.; Hu, Z.; Lin, Z.; Yang, T.; Lin, F.; Xing, Y.; Bai, J.; Sun, H.; Liang, Y. Chem. Mater. 2019, 31, 3025.

(105) Zhao, Q.; Xiao, Z.; Qu, J.; Liu, L.; Richter, H.; Chen, W.; Han, L.; Wang, M.; Zheng, J.; Xie, Z.; Ding, L.; He, F. ACS Energy Lett. 2019, 4, 1106.

(106) Zhan, L.; Li, S.; Zhang, S.; Chen, X.; Lau, T. K.; Lu, X.; Shi, M.; Li, C. Z.; Chen, H. ACS Appl. Mater. Interfaces 2018, 10, 42444.

(107) Xing, Z.; Meng, X.; Sun, R.; Hu, T.; Huang, Z.; Min, J.; Hu, X.; Chen, Y. Adv. Funct. Mater. 2020, 30, 2000417.

(108) Huang, W.; Jiang, Z.; Fukuda, K.; Jiao, X.; McNeill, C. R.; Yokota, T.; Someya, T. Joule 2020, 4, 128.

(109) Zhu, C.; Huang, H.; Jia, Z.; Cai, F.; Li, J.; Yuan, J.; Meng, L.; Peng, H.; Zhang, Z.; Zou, Y.; Li, Y. Sol. Energy 2020, 204, 660.

(110) Ha, J. W.; Song, C. E.; Kim, H. S.; Ryu, D. H.; Shin, W. S.; Hwang, D. H. ACS Appl. Mater. Interfaces 2020, 12, 51699.

(111) Szymanski, R.; Henry, R.; Stuard, S.; Vongsaysy, U.; Courtel, S.; Vellutini, L.; Bertrand, M.; Ade, H.; Chambon, S.; Wantz, G. Sol. RRL 2020, 4, 2000538.

(112) Andersen, T. R.; Zhao, F.; Li, Y.; Dickinson, M.; Chen, H. Sol. RRL 2020, 4, 2000246.

(113) Zhu, C.; Huang, H.; Jia, Z.; Cai, F.; Li, J.; Yuan, J.; Meng, L.; Peng, H.; Zhang, Z.; Zou, Y.; Li, Y. Sol. Energy 2020, 204, 660.

(114) Zhang, Y.; Liu, X.; Gu, H.; Yan, L.; Tan, H.; Ma, C.-Q.; Lin, Y. Org. Electron. 2020, 77, 105530.

(115) Hwang, H.; Park, C.; Sin, D. H.; Song, E.; Cho, K. Org. Electron. 2020, 83, 105738.

(116) Fan, B.; Zhong, W.; Jiang, X.-F.; Yin, Q.; Ying, L.; Huang, F.; Cao, Y. Adv. Energy Mater. 2017, 7, 1602127.

(117) Kim, M.; Lee, J.; Sin, D. H.; Lee, H.; Woo, H. Y.; Cho, K. ACS Appl. Mater. Interfaces 2018, 10, 25570.

(118) Lin, F.; Jiang, K.; Kaminsky, W.; Zhu, Z.; Jen, A. K. J. Am. Chem. Soc. 2020, 142, 15246.

(119) Li, J.; Liu, H.; Wang, Z.; Bai, Y.; Liu, L.; Wang, F.; Hayat, T.; Alsaedi, A.; Tan, Z. Macromol. Rapid Commun. 2018, 39, 1700492.

(120) Zhang, T.; Zhao, X.; Yang, D.; Tian, Y.; Yang, X. Adv. Energy Mater. 2018, 8, 1701691.

(121) Bi, P. Q.; Hall, C. R.; Yin, H.; So, S. K.; Smith, T. A.; Ghiggino, K. P.; Hao, X. T. J. Phys. Chem. C 2019, 123, 18294.

(122) Kranthiraja, K.; Aryal, U. K.; Sree, V. G.; Gunasekar, K.; Lee, C.; Kim, M.; Kim, B. J.; Song, M.; Jin, S. H. ACS Appl. Mater. Interfaces 2018, 10, 13748.

(123) Hwang, H.; Sin, D. H.; Park, C.; Cho, K. Sci. Rep. 2019, 9, 12081.

(124) Nam, M.; Yoo, J.; Park, Y.; Noh, H. Y.; Park, Y.; Cho, J.; Kim, J.-A.; Kim, J.; Lee, H. H.; Chang, R.; Ko, D.-H.J. Mater. Chem. A 2019, 7, 9698.

(125) Liu, F.; Li, C.; Li, J.; Wang, C.; Xiao, C.; Wu, Y.; Li, W. Chin. Chem. Lett. 2019, 31, 865.

(126) Zhong, W.; Cui, J.; Fan, B.; Ying, L.; Wang, Y.; Wang, X.; Zhang, G.; Jiang, X.-F.; Huang, F.; Cao, Y. Chem. Mater. 2017, $29,8177$.

(127) Sun, Y.; Li, G.; Wang, L.; Huai, Z.; Fan, R.; Huang, S.; Fu, G.; Yang, S. Sol. Energy Mater. Sol. Cells 2018, 182, 45.

(128) Nam, M.; Kang, J. h.; Shin, J.; Na, J.; Park, Y.; Cho, J.; Kim, B.; Lee, H. H.; Chang, R.; Ko, D. H. Adv. Energy Mater. 2019, 9, 1901856.

(129) Zhou, Z.; Xu, S.; Song, J.; Jin, Y.; Yue, Q.; Qian, Y.; Liu, F.; Zhang, F.; Zhu, X. Nat. Energy 2018, 3, 952. 
(130) Chen, Y.; Ye, P.; Zhu, Z. G.; Wang, X.; Yang, L.; Xu, X.; Wu, X.; Dong, T.; Zhang, H.; Hou, J.; Liu, F.; Huang, H. Adv. Mater. 2016, 29, 1603154

(131) Yin, P.; Wang, L.; Liang, J.; Yu, Y.; Chen, L.; Weng, C.; Cui, C.; Shen, P. J. Mater. Chem. C 2020, 8, 11223.

(132) Cheng, P.; Wang, R.; Zhu, J.; Huang, W.; Chang, S. Y.; Meng, L.; Sun, P.; Cheng, H. W.; Qin, M.; Zhu, C.; Zhan, X.; Yang, Y. Adv. Mater. 2018, 30, 1705243.

(133) Yin, P.; Zheng, T.; Wu, Y.; Liu, G.; Zhang, Z.-G.; Cui, C.; Li, Y.; Shen, P. J. Mater. Chem. A 2018, 6, 20313.

(134) Liu, T.; Xue, X.; Huo, L.; Sun, X.; An, Q.; Zhang, F.; Russell, T. P.; Liu, F.; Sun, Y. Chem. Mater. 2017, 29, 2914.

(135) Du, X.; Lu, X.; Zhao, J.; Zhang, Y.; Li, X.; Lin, H.; Zheng, C.; Tao, S. Adv. Funct. Mater. 2019, 29, 1902078.

(136) Sharapov, V.; Wu, Q.; Neshchadin, A.; Zhao, D.; Cai, Z.; Chen, W.; Yu, L. J. Phys. Chem. C 2018, 122, 11305.

(137) Duan, L.; Xu, C.; Yi, H.; Upama, M. B.; Mahmud, M. A.; Wang, D.; Haque, F.; Uddin, A. IEEE J. Photovoltaics 2019, 9, 1031.

(138) Zhang, H.; Wang, X.; Sun, Y.; Han, P.; Ren, J.; Sun, W.; Feng, S.; Ye, J.; Yang, S.; Bester, G.; Zhang, Y. J. Phys. D: Appl. Phys. 2020, 53, 095103.
(139) Fang, J.; Wang, Z.; Zhang, J.; Zhang, Y.; Deng, D.; Wang, Z.; Lu, K.; Ma, W.; Wei, Z. Adv. Sci. 2015, 2, 1500250.

(140) Zhang, M.; Zhu, L.; Zhou, G.; Hao, T.; Qiu, C.; Zhao, Z.; Hu, Q.; Larson, B. W.; Zhu, H.; Ma, Z.; Tang, Z.; Feng, W.; Zhang, Y.; Russell, T. P.; Liu, F. Nat. Commun. 2021, 12, 309.

(141) Jiang, K.; Zhang, J.; Peng, Z.; Lin, F.; Wu, S.; Li, Z.; Chen, Y.; Yan, H.; Ade, H.; Zhu, Z.; Jen, A. K. Nat. Commun. 2021, 12, 468.

(142) Zhu, Y.; Gadisa, A.; Peng, Z.; Ghasemi, M.; Ye, L.; Xu, Z.; Zhao, S.; Ade, H. Adv. Energy Mater. 2019, 9, 1900376.

(143) Liu, L.; Chao, P.; Mo, D.; He, F. J. Energy Chem. 2020, 54, 620.

(144) Privado, M.; Guijarro, F. G.; de la Cruz, P.; Singhal, R.; Langa, F.; Sharma, G. D. ACS Appl. Mater. Interfaces 2021, 13, 6461.

(145) Peng, Z.; Jiang, K.; Qin, Y.; Li, M.; Balar, N.; O'Connor, B. T.; Ade, H.; Ye, L.; Geng, Y. Adv. Energy Mater. 2021, 11, 2003506.

(146) Liu, Z.; Wang, N. Dyes Pigm. 2021, 187, 109111.

(147) Li, G.; Yang, T.; Cheng, H.; Zhang, Y.; Wang, J.; Liu, Y. Org. Electron. 2020, 87, 105904.

(148) Arunagiri, L.; Peng, Z.; Zou, X.; Yu, H.; Zhang, G.; Wang, Z.; Lin Lai, J. Y.; Zhang, J.; Zheng, Y.; Cui, C.; Huang, F.; Zou, Y.; Wong, K. S.; Chow, P. C. Y.; Ade, H.; Yan, H. Joule 2020, 4, 1790. 